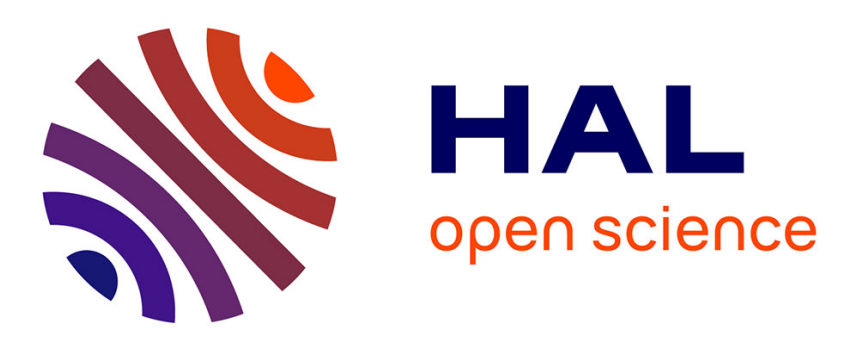

\title{
Information transfer between incompatible finite element meshes: application to coupled thermo-viscoelasticity
}

David Dureisseix, Henri Bavestrello

\section{To cite this version:}

David Dureisseix, Henri Bavestrello. Information transfer between incompatible finite element meshes: application to coupled thermo-viscoelasticity. Computer Methods in Applied Mechanics and Engineering, 2006, 195 (44-47), pp.6523-6541. 10.1016/j.cma.2006.02.003 . hal-00141161

\author{
HAL Id: hal-00141161 \\ https://hal.science/hal-00141161
}

Submitted on 1 Nov 2016

HAL is a multi-disciplinary open access archive for the deposit and dissemination of scientific research documents, whether they are published or not. The documents may come from teaching and research institutions in France or abroad, or from public or private research centers.
L'archive ouverte pluridisciplinaire HAL, est destinée au dépôt et à la diffusion de documents scientifiques de niveau recherche, publiés ou non, émanant des établissements d'enseignement et de recherche français ou étrangers, des laboratoires publics ou privés. 


\title{
Information transfer between incompatible finite element meshes: application to coupled thermo-viscoelasticity
}

\author{
David Dureisseix, Henri Bavestrello ${ }^{\dagger}$
}

\begin{abstract}
This article is concerned with information transfer between non-matching finite element meshes. Such a feature is not new in the literature, but we focus herein on a geometric approach to transfer solution fields in order to be as application independent as possible. Moreover, the dedicated case concerns the transfer of finite element fields defined at integration points of the meshes, and allows iterative exchange of fields in both directions. To do so, we propose an extension to the mortar technique that fulfills these goals and that does not suffer from a high computational cost. The application is the simulation of a strongly coupled thermo-viscoelastic problem with phase transition, solved with a partitioning technique.

This article in its final form was published in Computer Methods in Applied Mechanics and Engineering, Volume 195, Issues 44-47, 15 September 2006, Pages 6523-6541.

http://www.elsevier.com

doi:10.1016/j.cma.2006.02.003
\end{abstract}

keyword non-matching meshes, incompatible meshes, gluing, mortar, thermoviscoelasticity, coupled problem, partitioning strategy

PACS 65C20, 74S05, 65N55, 74D10

\section{Introduction}

The finite element method is an appreciable and wide spread tool for structural analysis, and, to a smaller extent, for fluid mechanics. The main feature of finite elements is the modularity in their usage, and an easy framework for integration (and so, a large applicability when the problem is settled with variational formulations). Once a mesh is produced, the solution fields of interest for the user are consistent and satisfy conservation principles, at least at the discretized level. Nevertheless, consideration of a second mesh of the same domain is often required. Among other examples, one can find the following applications:

\footnotetext{
*Corresponding author, LMGC, University Montpellier 2 / CNRS UMR 5508, CC 048, Place Eugène Bataillon, F-34095 MONTPELLIER CEDEX 5, FRANCE, David.Dureisseix@lmgc.univ-montp2.fr

${ }^{\dagger}$ Institute for Computational \& Mathematical Engineering, Stanford University, STANFORD, CA 94305, USA, Henri.Bavestrello@stanford.edu
} 
- where remeshing is required, in finite strain simulation to avoid distortion of elements (metal forming, machining...), when a crack propagates and the mesh is evolving accordingly (though alternative techniques are now available with quasi-fixed meshes), or when adaptive remeshing is due to an accuracy requirement;

- with different meshes for multiphysics simulation and staggered algorithms (thermo-mechanics with or without solidification, fluid-structure interaction in porous media...);

- or with specific applications with zooming techniques on a particular area (such as the Arlequin method [3, 4], global/local techniques [27, 39, 24], Chimera scheme $[37,36] \ldots)$

When dealing with incompatible (or non-matching) meshes, several situations lead to different cases; with an increasing order of difficulty, one may consider the following cases (Figure 1):

- identical meshes (obviously compatible) for which the information transfer operator should be identity;

- nested meshes (in fact, meshes for which the corresponding finite element spaces are nested). Multigrid techniques have been widely used in this case;

- non-nested meshes, but with a large difference in element size (coarse mesh and fine mesh), as this is often the case in aeroelasticity for instance;

- general case of dissimilar meshes, e.g. different mesh topologies and possibly different orders of approximation.

$\Omega_{1}$

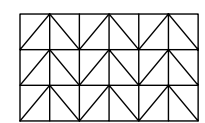

$\Omega_{2}$

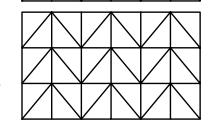

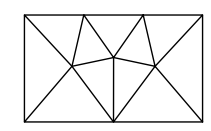

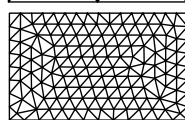

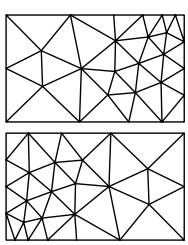

Figure 1: Sketch of different situations when comparing two meshes of the same domain (2D case). From left to right: compatible, nested, coarse and fine, dissimilar

Several existing approaches use refined procedures to enforce admissibility properties of the transferred field on the new mesh, as in [30,38] or as it can be done from [31]. Nevertheless, they often need an underlying transfer operation to 'geometrically' project the initial field.

At least, when transferring a nodal-based field, the interpolation of the field via finite element shape functions is available. When information is stored at integration points (stress, strain and internal variables in non-linear structural analysis, for instance), the situation is not so obvious.

In this article, we focus on solving coupled problems, like those arising from multiphysics models, where different meshes are used for different physics. Each 
of these meshes may have its own refinement requirements. To allow iterative solution strategies with a partitioning method (see [25, 16, 11] for instance), a tool for frequent transfers of material fields in both directions is required. Usually, the values of these fields are only available at integration points, as it is the case for the application developed herein: thermo-viscoelastic structure analysis.

After recalling two approaches for transferring nodal fields in the next Section, we focus on the case where the information is stored at integration points only, in Section 3. The choice of operators is discussed in order to satisfy two criteria: the patch test and the duality conservation. Section 4 deals with some implementation issues, and, finally, Section 5 presents an application with dissimilar meshes: the 2D simulation of strongly coupled thermo-viscoelasticity problems solved with a partitioning strategy. Comparison with a classical scheme, and performance evaluation in terms of precision are performed for problems with smooth or discontinuous solutions.

\section{Methods suited to nodal field transfer}

\subsection{Patch-test and collocation operators}

The simplest transfer operator consists of using the interpolation of a scalar field $e_{1}(M)$, lying on a first mesh $\Omega_{1}$, to compute its values at the nodes of a second mesh $\Omega_{2}$, to get the transferred field $e_{2}(M)$. Such a procedure will be called 'collocation'.

When considering a continuous nodal-valued field, if $\boldsymbol{N}_{1}(m)$ are the values at point $m$ (in the reference configuration) of the finite element shape functions, one gets $e_{1}(M)=\boldsymbol{N}_{1}(m) \boldsymbol{E}_{1} . \quad M$ is the current point where the value of the field $e_{1}(M)$ is searched (the nodes of mesh $\Omega_{2}$, see Figure 2, left), and $\boldsymbol{E}_{1}$ is the global nodal vector storing the values of the field. The corresponding prolongation operator (using multigrid terminology) is $\boldsymbol{P}_{21}^{c}$; it transfers a field from $\Omega_{1}$ to $\Omega_{2}: \boldsymbol{E}_{2}=\boldsymbol{P}_{21}^{c} \boldsymbol{E}_{1}$, and the transferred field is $e_{2}=\boldsymbol{N}_{2} \boldsymbol{E}_{2}$. Due to its definition, $\boldsymbol{P}_{21}^{c}$ only requires computation of the natural coordinates of the nodes of mesh $\Omega_{2}$ within the elements of the mesh $\Omega_{1}$.

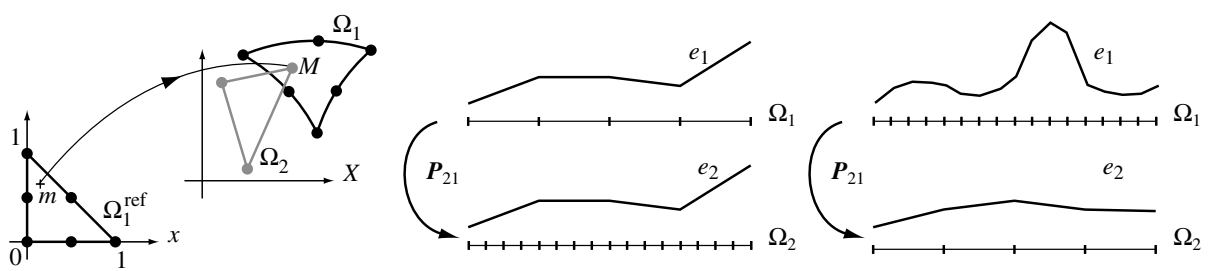

Figure 2: Finite element transformation from reference to real configuration (left) and examples of field transfer (middle and right)

This collocation operator is consistent, since if both meshes are identical, $\boldsymbol{P}_{21}^{c}$ is the identity operator. Moreover, if we are concerned with transfers in both directions, the couple of operators $\left(\boldsymbol{P}_{21}^{c}, \boldsymbol{P}_{12}^{c}\right)$ satisfies the patch test [41]: in the present situation, it concerns the accurate transmission of a field that can be 
exactly represented on both meshes (for instance, constant fields, or linear nodal fields, with respect to the coordinates of the mesh nodes, etc.) For the particular case where $\Omega_{2}$ is a fine mesh embedded into a coarse mesh $\Omega_{1}$, i.e. if the coarse finite element space in included into the fine one, the collocation operator $\boldsymbol{P}_{21}^{c}$ preserves the continuum fields (Figure 2, middle): $e_{2}=e_{1}, \boldsymbol{E}_{2}=\boldsymbol{P}_{21}^{c} \boldsymbol{E}_{1}$. This is the reason why this operator is also called a projector.

The drawback concerns the reciprocal operator $\boldsymbol{P}_{12}^{c}$ : it suffers from severe information loss when transferring from a fine mesh to a coarse one (Figure 2, right). In such a case, an averaging procedure seems more suitable, as classically done for the fluid pressure in aeroelasticity for instance $[28,23,14]$. Such an averaging procedure is described in the following Section.

\subsection{Duality conservation and mortar operators}

\subsubsection{Generalized average}

To derive a generalized averaging approach without smoothing (a discussion on this point is postponed to Section 5.4.1) we propose herein to rely on the conservation of work for two dual fields, each being subjected to be transferred in one direction.

In order to transfer the field $e_{1}$ to the mesh $\Omega_{2}$, the first step is to consider a symmetric form acting both on this field and on a dual field, denoted with $f_{1}$, to get the energy $<e_{1}, f_{1}>_{\Omega_{1}}$. We choose to express the dual field with its interpolable form $f_{1}=\boldsymbol{N}_{1} \boldsymbol{F}_{1}$ :

$$
<e_{1}, f_{1}>_{\Omega_{1}}=\int_{\Omega_{1}} e_{1} \cdot f_{1} d \Omega_{1}=\boldsymbol{E}_{1}^{T}\left(\int_{\Omega_{1}} \boldsymbol{N}_{1}^{T} \boldsymbol{N}_{1} d \Omega_{1}\right) \boldsymbol{F}_{1}=\boldsymbol{E}_{1}^{T} \boldsymbol{M}_{1} \boldsymbol{F}_{1}
$$

Superscript $T$ denotes the $L_{2}$ transposition, and $M_{1}$ is the integral of the cross product of shape functions. It is abusively called the "mass" matrix (actually, no physical mass density is involved); it is symmetric and positive definite (SPD, as long as no numerical under-integration is used), and therefore invertible.

The second step is to express the conservation of the work between the two meshes:

$$
\int_{\Omega_{1}} e_{1} \cdot f_{1} d \Omega_{1}=\int_{\Omega_{2}} e_{2} \cdot f_{2} d \Omega_{2}
$$

This property is explicitly used in a large number of multiscale approaches (see multigrid methods and [21] for instance). Using the nodal values of the fields, one gets: $\boldsymbol{E}_{1}^{T} \boldsymbol{M}_{1} \boldsymbol{F}_{1}=\boldsymbol{E}_{2}^{T} \boldsymbol{M}_{2} \boldsymbol{F}_{2}$. If we recall that $\boldsymbol{E}_{2}=\boldsymbol{P}_{21} \boldsymbol{E}_{1}$, enforcing this condition for any field $\boldsymbol{E}_{1}$ leads to:

$$
\boldsymbol{F}_{1}=\boldsymbol{M}_{1}^{-1} \boldsymbol{P}_{21}^{T} \boldsymbol{M}_{2} \boldsymbol{F}_{2}=\boldsymbol{R}_{12} \boldsymbol{F}_{2}
$$

The restriction operator (with the multigrid terminology) $\boldsymbol{R}_{12}$ is an average selection using coarse shape functions, and the dual of the prolongation with respect to the $\boldsymbol{M}$-dot product:

$$
\boldsymbol{R}_{12}=\boldsymbol{M}_{1}^{-1} \boldsymbol{P}_{21}^{T} \boldsymbol{M}_{2} \quad \text { and } \quad \boldsymbol{P}_{21}=\boldsymbol{M}_{2}^{-1} \boldsymbol{R}_{12}^{T} \boldsymbol{M}_{1}
$$

Note that this does not select the couple $\left(\boldsymbol{P}_{21}, \boldsymbol{R}_{12}\right)$ but establishes the consistent relationship between them. Choosing $\boldsymbol{P}_{21}=\boldsymbol{P}_{21}^{c}$ as a collocation operator, 
and $\boldsymbol{P}_{12}=\boldsymbol{R}_{12}$ fixed the aforementioned drawback. But this new couple of operators does not satisfy the patch test anymore, except for the special case of embedded meshes, when $\Omega_{2}$ is the fine mesh, and $\Omega_{1}$ the coarse one. To recover the patch-test verification, one can use the so-called mortar operators.

\subsubsection{Mortar projection}

Briefly, the way the mortar approach $[5,6,1,2]$ proceeds to transfer the field $e_{1}=\boldsymbol{N}_{1} \boldsymbol{E}_{1}$ to the field $e_{2}=\boldsymbol{N}_{2} \boldsymbol{E}_{2}$ is to express the equivalence of the generalized averages of both fields with respect to the weight functions $\boldsymbol{N}_{2}$ :

$$
\int_{\Omega_{2}} \boldsymbol{N}_{2}^{T} e_{2} d \Omega_{2}=\int_{\Omega_{1}} \boldsymbol{N}_{2}^{T} e_{1} d \Omega_{1}
$$

It leads to

$$
\boldsymbol{M}_{2} \boldsymbol{E}_{2}=\int_{\Omega_{1}} \boldsymbol{N}_{2}^{T} \boldsymbol{N}_{1} d \Omega_{1} \boldsymbol{E}_{1}=\boldsymbol{M}_{21} \boldsymbol{E}_{1}
$$

$\boldsymbol{M}_{12}$ denotes the integral of the cross product of shape functions $\boldsymbol{N}_{1}$ and $\boldsymbol{N}_{2}$, arising from the different meshes. Then, $\boldsymbol{E}_{2}=\boldsymbol{P}_{21} \boldsymbol{E}_{1}$ with $\boldsymbol{P}_{21}=\boldsymbol{M}_{2}^{-1} \boldsymbol{M}_{21}$ which is known as the mortar projector. With the previous consistent relationship, one gets the symmetric expression $\boldsymbol{R}_{12}=\boldsymbol{M}_{1}^{-1} \boldsymbol{M}_{12}$.

An appealing property is the ability of this approach to satisfy the patch test for dissimilar meshes. Effectively, if a field $e$ is representable on both meshes, i.e. if $e=\boldsymbol{N}_{1} \boldsymbol{E}_{1}=\boldsymbol{N}_{2} \boldsymbol{E}_{2}$, transferring $\boldsymbol{E}_{1}$ to the mesh $\Omega_{2}$ leads to:

$$
\begin{aligned}
\boldsymbol{P}_{21} \boldsymbol{E}_{1}=\boldsymbol{M}_{2}^{-1} \boldsymbol{M}_{21} \boldsymbol{E}_{1}=\boldsymbol{M}_{2}^{-1} \int_{\Omega_{2}} \boldsymbol{N}_{2}^{T} \boldsymbol{N}_{1} \boldsymbol{E}_{1} d \Omega_{2}= \\
\boldsymbol{M}_{2}^{-1} \int_{\Omega_{2}} \boldsymbol{N}_{2}^{T} \boldsymbol{N}_{2} \boldsymbol{E}_{2} d \Omega_{2}=\boldsymbol{M}_{2}^{-1} \boldsymbol{M}_{2} \boldsymbol{E}_{2}
\end{aligned}
$$

Therefore, one effectively gets $\boldsymbol{P}_{21} \boldsymbol{E}_{1}=\boldsymbol{E}_{2}$. With the symmetry of the formulation, the transfer of dual quantities with the dual operator leads to the same result. Therefore, the mortar operators satisfy the patch test. These operators are also projectors, because in the case of nested meshes, they are identical to collocation projectors. As a corollary, they are also consistent (they lead to the identity for identical meshes).

The drawbacks are, as previously, the need for global mass matrices with explicit inversion for a direct method, or global resolutions (factorization, forward and backward substitutions) for an iterative solution algorithm, as well as the construction of integral cross products of incompatible shape functions.

\section{Case of sampled field transfer}

Dealing with 'sampled' fields defined at integration points is a more complicated situation $[32,33,35,34,29]$. The problem is now to transfer information from a set of values at integration points of a mesh $\Omega_{1}$ to a set of values at integration points of a dissimilar mesh $\Omega_{2}$.

If $\mathcal{E}$ denotes the column vector of values of a field $e$ at integration points $M_{k}$ of a mesh $\Omega$, the integration on this mesh uses the approximate evaluation of 
integrals using the values of the jacobian of the transformation at those points, as well as the so-called weights $w_{k}$ of those points [42]:

$$
\int_{\Omega} e \cdot f d \Omega \approx \sum_{\Omega_{\mathrm{el}} \in \Omega} \sum_{k=1}^{n_{\mathrm{el}}^{i}} e\left(M_{k}\right) f\left(M_{k}\right) w_{k} J\left(M_{k}\right)=\mathcal{E}^{T} \boldsymbol{W} \mathcal{F}
$$

The approximation involves the sum on each element $\Omega_{\mathrm{el}}$ of the mesh $\Omega$, and on each integration point $k$ of each element. $n_{\mathrm{el}}^{i}$ denotes the number of integration points within the element $\Omega_{\mathrm{el}}$. Several local integration schemes are available, such as Gauss quadrature, Newton-Cotes, Romberg, Gauss-Kronrod... [9, 42].

In each case, they involve a diagonal matrix $\boldsymbol{W}$ storing the integration information. $\mathcal{F}$ is of course the column vector of dual field values at integration points.

The same requirement of duality conservation leads to the same kind of relationship between a prolongation $\mathcal{P}_{21}$ defined between integration points of the meshes, and the restriction $\mathcal{R}_{12}$ :

$$
\boldsymbol{\mathcal { R }}_{12}=\boldsymbol{W}_{1}^{-1} \mathcal{P}_{21}^{T} \boldsymbol{W}_{2} \quad \text { or } \quad \mathcal{P}_{21}=\boldsymbol{W}_{2}^{-1} \mathcal{R}_{12}^{T} \boldsymbol{W}_{1}
$$

The choice of these operators is not obvious; this is the goal of the following developments.

To transfer a sampled field to another mesh or to other points, a kind of extrapolation is necessary. The simplest proposal (but not the sole one, see for instance [38]) is to use the shape functions on each element independently: the sampled field on each element (defined at integration points) must then be extrapolated to the nodes of the element in order to be later interpolated with shape functions. This procedure is done at the element level, and so, the extrapolated field is discontinuous throughout element edges. Its values are given at each node of each element; on a given node, there will be as many values as there are elements connected to this node. The overall procedure is split into several steps:

- the sampled field given at integration points of a first mesh is extrapolated at the element level to the nodes of the same mesh. This results in an interpolable field, which is a priori discontinuous throughout the element edges;

- this last field is transferred to the second mesh via a mortar-like procedure. The resulting field lies on the second mesh and is still discontinuous;

- this field is finally interpolated at element level to the integration points of this last mesh.

\subsection{Choice of elemental operators}

A sampled field $\mathcal{E}$ is extrapolated, using a linear operator $\mathcal{L}$, to a field $\boldsymbol{E}$ defined at the nodes of the element: $\boldsymbol{E}=\mathcal{L} \mathcal{E}$. With the same framework as in the previous Section, the dual field, $\boldsymbol{F}$, is interpolated at integration points with an interpolation operator $\mathcal{N}: \mathcal{F}=\mathcal{N} \boldsymbol{F}$. This is shown in Figure 3, where $\Omega^{i}$ denotes the set of integration points, while $\Omega^{o}$ is the set of nodes, both are defined for each element independently. 
The same requirement holds, i.e. the duality should be preserved:

$$
\mathcal{E}^{T} \boldsymbol{W} \mathcal{F}=\boldsymbol{E}^{T} \boldsymbol{M F}
$$

This time, all quantities are defined at the element level, so the integration operator $\boldsymbol{W}$ and the elementary mass matrix $\boldsymbol{M}$ are small in size. The same conclusion holds: interpolation and extrapolation should not be chosen independently, but must satisfy the relationship (at element level):

$$
\mathcal{L}=\boldsymbol{M}^{-1} \mathcal{N}^{T} \boldsymbol{W} \quad \text { or } \quad \mathcal{N}=\boldsymbol{W}^{-1} \mathcal{L}^{T} \boldsymbol{M}
$$

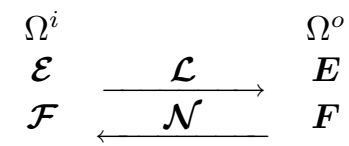

Figure 3: Extrapolation and interpolation on the same mesh

Notice that all matrices are defined at the element level, and so, are small in size.

At this point, several possibilities exist for extrapolation and interpolation. To choose them, let us first have a glance at the construction of the elementary 'mass' matrix. On the element $\Omega_{\mathrm{el}}$, when acting on two fields $\boldsymbol{E}$ and $\boldsymbol{E}^{\prime}$, it is defined as:

$$
\begin{aligned}
\boldsymbol{E}^{T} \boldsymbol{M} \boldsymbol{E}^{\prime}=\int_{\Omega_{\mathrm{el}}} e \cdot e^{\prime} d \Omega_{\mathrm{el}}=\boldsymbol{E}^{T} \int_{\Omega_{\mathrm{el}}} \boldsymbol{N}^{T} \boldsymbol{N} d \Omega_{\mathrm{el}} \boldsymbol{E}^{\prime} \approx \\
\boldsymbol{E}^{T}\left(\sum_{k=1}^{n_{\mathrm{el}}^{i}} \boldsymbol{N}\left(M_{k}\right)^{T} \boldsymbol{N}\left(M_{k}\right) w_{k} J\left(M_{k}\right)\right) \boldsymbol{E}^{\prime}
\end{aligned}
$$

Therefore, if we choose the interpolation $\mathcal{N}$ to store the values of shape functions at integration points, $\boldsymbol{N}\left(M_{k}\right)$, we get $\boldsymbol{M}=\mathcal{N}^{T} \boldsymbol{W} \mathcal{N}$ and the equivalence in energy: $\boldsymbol{E}^{T} \boldsymbol{M} \boldsymbol{E}^{\prime}=\mathcal{E}^{T} \boldsymbol{W} \mathcal{E}^{\prime}$, with $\mathcal{E}$ and $\mathcal{E}^{\prime}$ as interpolated fields from $\boldsymbol{E}$ and $\boldsymbol{E}^{\prime}$.

Once the interpolation is chosen, the conservation of duality (5) leads to the associated extrapolation: $\mathcal{L}=\boldsymbol{M}^{-1} \mathcal{N}^{T} \boldsymbol{W}$. All of these matrices are defined at the element level only.

An interpretation of the extrapolation is the following: once the values at integration points $\mathcal{E}$ are given, one gets the nodal values $\boldsymbol{E}$ of a interpolable finite element field whose values at the same integration points, $\mathcal{N} \boldsymbol{E}$, minimize the distance:

$$
\min _{\boldsymbol{E}} \frac{1}{2}(\mathcal{N} \boldsymbol{E}-\mathcal{E})^{T} \boldsymbol{W}(\mathcal{N} \boldsymbol{E}-\mathcal{E})
$$

leading to: $\mathcal{N}^{T} \boldsymbol{W} \mathcal{N} \boldsymbol{E}=\mathcal{N}^{T} \boldsymbol{W} \mathcal{E}$ and so $\boldsymbol{M E}=\boldsymbol{M}^{-1} \mathcal{N}^{T} \boldsymbol{W} \mathcal{E}=\mathcal{L} \mathcal{E}$. A direct consequence of the previous choice of interpolation and extrapolation is the property:

$$
\mathcal{L} \mathcal{N}=\boldsymbol{M}^{-1} \mathcal{N}^{T} \boldsymbol{W} \mathcal{N}=\boldsymbol{M}^{-1} \boldsymbol{M}=\mathbf{1}_{o}
$$

where $\mathbf{1}_{o}$ is the identity matrix on the element-wise nodal fields. This property simply states that if a nodal field is interpolated at integration points, then the 
back-extrapolation at nodes will restore the original field. One can remark that this is only possible if the number of integration points $n_{\mathrm{el}}^{i}$ is at least equal to the number of nodes $n_{\mathrm{el}}^{o}$. This condition was hidden in the requirement for the computed elementary mass matrix to be invertible: suppose that all integration points have positive weight, and that the element is not too distorted (no hourglass configuration), then, $\boldsymbol{W}$ is positive definite (as well as diagonal). Thus for $\boldsymbol{M}=\mathcal{N}^{T} \boldsymbol{W} \mathcal{N}$ to be invertible, $\mathcal{N}$ should be injective, and so a necessary condition is $n_{\mathrm{el}}^{i} \geq n_{\mathrm{el}}^{o}$.

If it is not the case, $\boldsymbol{M}$ is under-integrated and singular. This particular situation will be discussed in the appendix A.

\subsection{A discontinuous mortar-like approach}

Once the interpolation and extrapolation are chosen, the next step is to give the prolongation and restriction of generated fields at element level. The overall sketch is depicted on Figure 4.

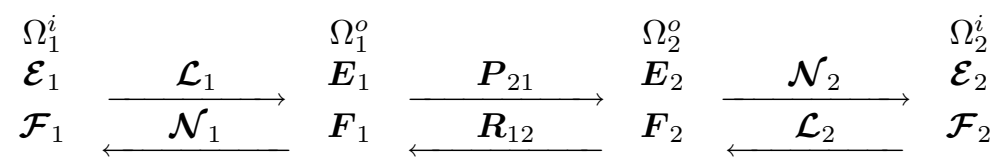

Figure 4: Prolongation and restriction for sampled fields

The underlying prolongation is chosen as the elementary mortar: $\boldsymbol{P}_{21}=$ $\boldsymbol{M}_{2}^{-1} \boldsymbol{M}_{21}$ (with all elementary matrices). With the conservation of duality, the restriction is:

$$
\boldsymbol{R}_{12}=\boldsymbol{M}_{1}^{-1} \boldsymbol{P}_{21}^{T} \boldsymbol{M}_{2}=\boldsymbol{M}_{1}^{-1} \boldsymbol{M}_{21}^{T}
$$

Of course, with all these operators, one gets automatically the duality conservation for the overall restriction and prolongation:

$$
\boldsymbol{\mathcal { R }}_{12}=\boldsymbol{W}_{1}^{-1} \mathcal{P}_{21}^{T} \boldsymbol{W}_{2} \quad \text { with } \quad \mathcal{P}_{21}=\mathcal{N}_{2} \boldsymbol{P}_{21} \mathcal{L}_{1} \quad \text { and } \quad \boldsymbol{R}_{12}=\mathcal{N}_{1} \boldsymbol{R}_{12} \mathcal{L}_{2}
$$

Referring to the classification of transfer approaches suggested in [29], the one proposed herein falls within the category of 'weak enforcement continuity transfers', as opposed to 'variationally consistent' and 'smoothing' transfers.

\subsection{Symmetry of the formulation}

Consider now the reverse transfer operation: transferring from mesh $\Omega_{2}$ to mesh $\Omega_{1}$. $\mathcal{P}_{12}$ and $\boldsymbol{\mathcal { R }}_{21}$ are defined in a similar way as already done for $\boldsymbol{P}_{21}$ and $\boldsymbol{\mathcal { R }}_{12}$.

One gets $\mathcal{R}_{21}=\boldsymbol{W}_{2}^{-1} \mathcal{P}_{12}^{T} \boldsymbol{W}_{1}=\boldsymbol{W}_{2}^{-1}\left(\mathcal{N}_{1} \boldsymbol{P}_{12} \mathcal{L}_{2}\right)^{T} \boldsymbol{W}_{1}$. Together with $\boldsymbol{W}_{2}^{-1} \mathcal{L}_{2}^{T}=\mathcal{N}_{2} \boldsymbol{M}_{2}^{-1}$ and $\boldsymbol{P}_{12}=\boldsymbol{M}_{1}^{-1} \boldsymbol{M}_{12}$, some algebraic manipulations lead to $\mathcal{R}_{21}=\mathcal{N}_{2} \boldsymbol{M}_{2}^{-1} \boldsymbol{M}_{12}^{T} \mathcal{L}_{1}$. If $\boldsymbol{M}_{12}^{T}=\boldsymbol{M}_{21}$, which is trivially satisfied for conforming discretized geometry, one gets $\boldsymbol{\mathcal { R }}_{21}=\mathcal{P}_{21}$. A similar argument gives $\boldsymbol{\mathcal { R }}_{12}=\mathcal{P}_{12}$

\subsection{Duality conservation and homogenization}

One can notice that the proposed transfer operators do not preserve the root mean square (RMS) value of a field, i.e. if $e_{2}$ is projected from $e_{1}$, the following 
is not satisfied in general:

$$
\int_{\Omega_{1}} e_{1}^{2} d \Omega_{1}=\int_{\Omega_{2}} e_{2}^{2} d \Omega_{2} \quad \text { or } \quad \boldsymbol{E}_{1}^{T} \boldsymbol{M}_{1} \boldsymbol{E}_{1}=\boldsymbol{E}_{2}^{T} \boldsymbol{M}_{2} \boldsymbol{E}_{2}
$$

Several remarks can be stated:

- this is not a requirement on the energy of the transferred field: to be so, it would be necessary to introduce a constitutive relation between one field and its dual counterpart;

- such an equivalence is not sufficient for defining a transfer operator. Consider for instance a model $1 \mathrm{D}$ problem with a field $e_{1}$ on a fine mesh that has to be transferred to a field $e_{2}$ on a coarse mesh such that $e_{2}$ has to be constant over an element. If $e_{1}$ possess a null standard average, as in Figure 5, two opposite, and non null, fields $e_{2}$ satisfy (8), and none are satisfactory.

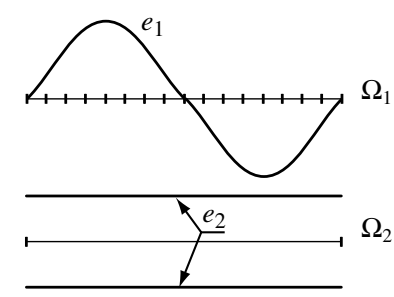

Figure 5: Transfer from fine mesh to coarse mesh with RMS conservation

The proposed approach deals with a dual couple of fields $\left(e_{1}, f_{1}\right)$ and consistency of projections in both ways is enforced. This approach is close to homogenization: if the relationship between the quantity $e_{1}$ and its dual counterpart $f_{1}$ is performed through a constitutive behavior operator (for the linear case, it can be expressed as: $\left.f_{1}=D e_{1}\right)$, the corresponding energy on $\Omega_{1}$ is

$$
\frac{1}{2} \int_{\Omega_{1}} e_{1} \cdot D e_{1} d \Omega_{1}=\frac{1}{2} \boldsymbol{E}_{1}^{T} \boldsymbol{K}_{11} \boldsymbol{E}_{1} d \Omega_{1}
$$

where $\boldsymbol{K}_{11}$ is the corresponding discretized operator (stiffness matrix for elasticity, for instance). On $\Omega_{2}$, it is

$$
\frac{1}{2} \int_{\Omega_{2}} e_{2} \cdot D e_{2} d \Omega_{2}=\frac{1}{2} \boldsymbol{E}_{2}^{T} \boldsymbol{K}_{22} \boldsymbol{E}_{2} d \Omega_{2}=\frac{1}{2} \boldsymbol{E}_{1}^{T} \boldsymbol{P}_{21}^{T} \boldsymbol{K}_{22} \boldsymbol{P}_{21} \boldsymbol{E}_{1}
$$

For conforming geometry, there will be duality conservation only if $\boldsymbol{K}_{11}=$ $\boldsymbol{P}_{21}^{T} \boldsymbol{K}_{22} \boldsymbol{P}_{21}$. It is an homogenization equivalence condition that gives the macroscopic behavior $\boldsymbol{K}_{11}$, from microscopic local behavior $\boldsymbol{K}_{22}$ (with the Voigt method if $e_{1}$ is a kinematic quantity; with the Reuss method if $e_{1}$ is a static quantity). $\boldsymbol{P}_{21}$ is the localization operator. This interpretation is valid only if $\Omega_{2}$ is a fine mesh, and $\Omega_{1}$ a coarse mesh. If the meshes are nested, this corresponds to the average law (or inverse average law depending on the choice 
of field $e_{1}$ ); otherwise, it leads to its discretized version that take into account the mesh incompatibility of finite elements. For the general case of different meshes, the link between discretized behavior operators is $\boldsymbol{K}_{11}=\boldsymbol{R}_{12} \boldsymbol{K}_{22} \boldsymbol{P}_{21}$ and $\boldsymbol{K}_{22}=\boldsymbol{R}_{21} \boldsymbol{K}_{11} \boldsymbol{P}_{12}$.

\section{Extensions and implementation issues}

\subsection{Extension to vector or tensor field transfer}

The proposed approach has been designed for scalar field transfers in the previous Sections. The extension to the vector or tensor case is immediate as soon as we state that the energy is a linear combination of products of dual field scalar components. For instance, let us consider the case of a stress tensor $\boldsymbol{\sigma}=\sum_{i, j} \sigma_{i j} n_{i} \otimes n_{j}$, and a dual strain tensor $\varepsilon=\sum_{i, j} \varepsilon_{i j} n_{i} \otimes n_{j}$, where $n_{i}$ is the basis unit vector number $i$. The corresponding symmetric form is

$$
\int_{\Omega} \operatorname{Tr}[\boldsymbol{\sigma} \varepsilon] d \Omega=\sum_{i, j} \int_{\Omega} \sigma_{i j} \varepsilon_{i j} d \Omega
$$

When the information exchange is required between two meshes $\Omega_{1}$ and $\Omega_{2}$, the duality conservation for any couple of fields requires the conservation for each component:

$$
\forall i, j \quad \int_{\Omega_{1}} \sigma_{i j}^{(1)} \varepsilon_{i j}^{(1)} d \Omega_{1}=\int_{\Omega_{2}} \sigma_{i j}^{(2)} \varepsilon_{i j}^{(2)} d \Omega_{2}
$$

Therefore, the scalar transfer operator should be applied to each component to define the transfer of the whole tensor (or vector) field. This will ensure the duality conservation as well.

\subsection{Integration issues}

Concerning implementation, the main difficulty is the evaluation of the integral of shape function cross product $\boldsymbol{M}_{21}$. This is performed at the element level with the following approach:

- neighboring elements of the two meshes $\Omega_{1}$ and $\Omega_{2}$ are scanned with a 2-level search (potential neighbors are selected with a box intersection test);

- for each couple of potential neighbors, the intersection of the elements is meshed; this is a classical problem in Computational Geometry. For 2D problems, this is related to the intersection of convex polygons, and we used herein the algorithm proposed in [19]. 3D problems are more CPU consuming, but other efficient approaches are also available, see [18] for instance;

- the 'mass' matrix of the intersecting mesh is computed as $\boldsymbol{M}_{3}$. Since an intersecting mesh (numbered as 3) is nested within each of the elements of the initial meshes, the local collocation operators $\boldsymbol{P}_{31}^{c}$ and $\boldsymbol{P}_{32}^{c}$ are computed (notice that they are the one-way transfer from a coarse mesh, 
$\Omega_{1}$ or $\Omega_{2}$, embedded into a finer one $\left.\Omega_{3}\right)$. The elementary contribution of the element 2 to the element 1 is $\boldsymbol{M}_{12}^{e}=\left(\boldsymbol{P}_{31}^{c}\right)^{T} \boldsymbol{M}_{3} \boldsymbol{P}_{32}^{c}$. All the neighbors of element 1 contributed to $\boldsymbol{M}_{12}$. The corresponding contribution to the element 2 is $\boldsymbol{M}_{21}^{e}=\left(\boldsymbol{M}_{12}^{e}\right)^{T}$.

\subsection{Specific features of the mortar approach}

The connectivity between integration points of different meshes can be large because its involves connected elements on each meshes. This can be seen on the bandwidth of matrices $\boldsymbol{P}_{21}$ and $\boldsymbol{R}_{12}$ if they have to be assembled. This connectivity can be decreased by using the dual mortar shape functions [40]. Such a feature is not useful herein since all the transfer operators are defined at element level. Moreover, they do not need to be assembled when using a partitioning solution strategy, which is the case we are focussing on, as illustrated in the applications of Section 5.

Concerning non-matching geometries, even if the continuum geometry is the same for the two discretizations, the discretized geometries can be not exactly compatible (as for the rotating disk example of the next Section): the discretized boundaries do not match exactly (Figure 6).
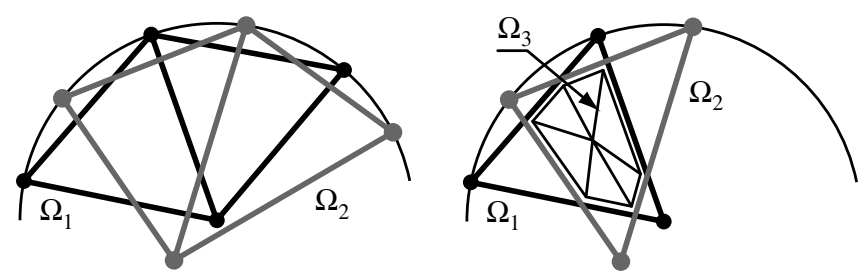

Figure 6: Non-matching discretized geometry and corresponding integration

For such cases the patch test is no more satisfied exactly. This can cause a problem for accuracy, especially when non null values are prescribed on a non conforming boundary. Special treatment of shape functions connected to nodes on the boundary is provided in the classical mortar approach but it leads to complex adaptations in 2D and 3D [8, 40,20]. Herein, a much simpler adaptation is available, an discussed in the following. Let us consider the overall intersecting mesh $\Omega_{3}$ (assembly of all element-element intersections, though not explicitly built in the implementation). The adaptation we use consists in expressing the integration over $\Omega_{3}$ rather than on $\Omega_{1}$ and $\Omega_{2}$. In particular, the duality conservation (1) becomes:

$$
\int_{\Omega_{3}} e_{1} \cdot f_{1} d \Omega_{3}=\int_{\Omega_{3}} e_{2} \cdot f_{2} d \Omega_{3}
$$

Clearly, this recovers the particular case of compatible geometry. For non compatible geometries, the only change in implementation is the computation of the elementary mass matrices as the assembly of $\left(\boldsymbol{P}_{31}^{c}\right)^{T} \boldsymbol{M}_{3} \boldsymbol{P}_{31}^{c}$ for $\boldsymbol{M}_{1}$, and $\left(\boldsymbol{P}_{32}^{c}\right)^{T} \boldsymbol{M}_{3} \boldsymbol{P}_{32}^{c}$ for $\boldsymbol{M}_{2}$. Therefore, if we consider a field such that $\boldsymbol{N}_{1} \boldsymbol{E}_{1}=\boldsymbol{N}_{2} \boldsymbol{E}_{2}$ anywhere on $\Omega_{1} \cap \Omega_{2}$, the proposed adaptation allows to have $\boldsymbol{M}_{21} \boldsymbol{E}_{1}=\boldsymbol{M}_{2} \boldsymbol{E}_{2}$. Finally, with $\mathcal{E}_{1}=\mathcal{N}_{1} \boldsymbol{E}_{1}$ and $\mathcal{E}_{2}=\mathcal{N}_{2} \boldsymbol{E}_{2}$, one gets, using 
(6),

$$
\begin{aligned}
\mathcal{P}_{21} \mathcal{E}_{1}=\mathcal{N}_{2} \boldsymbol{P}_{21} \mathcal{L}_{1} \mathcal{E}_{1}=\mathcal{N}_{2} \boldsymbol{P}_{21} \mathcal{L}_{1} \mathcal{N}_{1} \boldsymbol{E}_{1}=\mathcal{N}_{2} \boldsymbol{P}_{21} \boldsymbol{E}_{1}= \\
\mathcal{N}_{2} \boldsymbol{M}_{2}^{-1} \boldsymbol{M}_{21} \boldsymbol{E}_{1}=\mathcal{N}_{2} \boldsymbol{M}_{2}^{-1} \boldsymbol{M}_{2} \boldsymbol{E}_{2}=\mathcal{E}_{2}
\end{aligned}
$$

Since we get $\mathcal{P}_{21} \mathcal{E}_{1}=\mathcal{E}_{2}$ the patch test is satisfied again.

\subsection{A first test for one-way transfers: recursive remapping on a rotating mesh}

In order to test the transfer operation, in only one way, we use the test of the recursive remapping on a rotating mesh proposed in [34]: we recursively applying the transfer operation to a circular mesh, with unitary radius, which is rotated in a series of steps. The field to be transferred is given by the values at the integration points of the function $f(r, \theta)=16 r^{2}(1-r)^{2}|\cos 2 \theta| . r \in[0,1]$ and $\theta \in[0,2 \pi]$ are the polar coordinates of the integration points, and the maximum value of the function $f$ is 1 , Figure 7 .

The mesh is then rotated up to $\frac{\pi}{2}$ in a series of $N$ steps, the considered field at integration points is transferred from one mesh to the next one after each increment of rotation. As the function $f$ and the disk are invariant with respect to $\frac{\pi}{2}$ rotations, an exact transfer operation would reproduce the original function after $N$ projection steps. The deviation from this ideal case is measured with the error

$$
e_{N}=\left[\int_{S}\left(f-f_{N}\right)^{2} \frac{d S}{S}\right]^{1 / 2}
$$

where $f_{N}$ is the field obtained after $N$ projections.

The upper and lower parts of the disk are meshed herein with different element sizes: the element size ratio is about 3 . The goal is to test in the same shot the transfers between meshes with (i) comparable element sizes (fine or coarse meshes), (ii) from small sized elements to larger ones and (iii) the reversed situation. All these situations are encountered in the same test, provided that the integration domain $S$ of the error is chosen to be the appropriate quadrant of the disk (I, II, III or IV, see Figure 7).
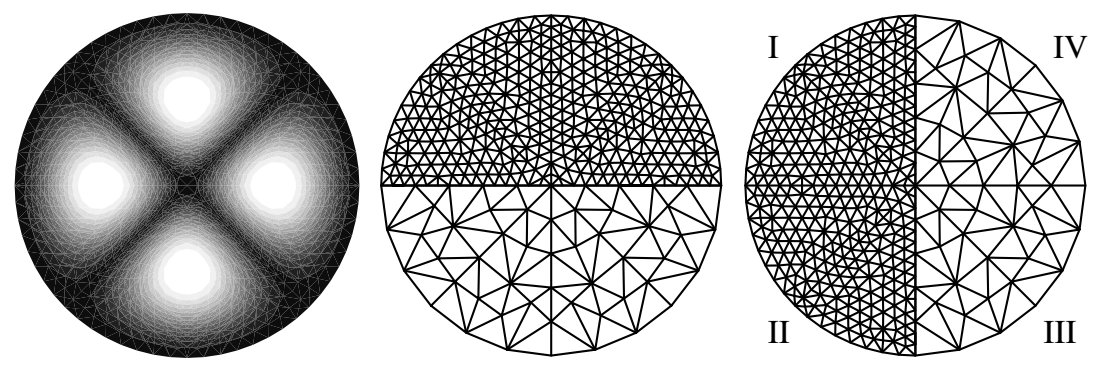

Figure 7: Initial field (left) with the initial (middle) and final (right) meshes of the four quadrants for the rotating disk test

As a comparison point of view, the results obtained in [34] are recalled within parenthesis together with the results obtained with the proposed approach, Ta- 


\begin{tabular}{|c|c|c|c|c|}
\hline$N$ & $\begin{array}{c}e_{N}^{I} \%(\star) \\
\text { (fine to fine) }\end{array}$ & $\begin{array}{c}e_{N}^{I I} \% \\
\text { (fine to coarse) }\end{array}$ & $\begin{array}{c}e_{N}^{I I} \% \\
\text { (coarse to coarse) }\end{array}$ & $\begin{array}{c}e_{N}^{I V} \% \\
\text { (coarse to fine) }\end{array}$ \\
\hline 4 & $0.506(0.360)$ & 1.887 & 1.377 & 1.014 \\
\hline 5 & $0.559(0.330)$ & 2.740 & 2.326 & 1.808 \\
\hline 6 & $0.530(0.396)$ & 2.599 & 2.773 & 1.987 \\
\hline 7 & $0.580(0.816)$ & 2.710 & 2.977 & 2.117 \\
\hline 8 & $0.632(0.426)$ & 2.869 & 3.153 & 2.241 \\
\hline 9 & $0.706(0.990)$ & 2.909 & 3.331 & 2.341 \\
\hline 10 & $0.706(0.791)$ & 2.994 & 3.492 & 2.477 \\
\hline 11 & $0.751(0.565)$ & 3.052 & 3.631 & 2.579 \\
\hline 12 & $0.789(1.061)$ & 3.124 & 3.753 & 2.678 \\
\hline
\end{tabular}

Table 1: Comparative errors obtained with rotating disk

ble 1. Note that the mesh is not the same as the one used in this reference, which was a block structured quadrilateral meshing of the disk, but the element size was comparable with the one of the present fine mesh (704 quadrilateral elements in the former reference for the whole disk, while 664 triangular elements are used herein for the fine meshed half part of the disk; the coarse mesh of the second part uses 84 elements). Therefore, one can only compare the order of magnitude of the errors between the aforementioned test and the present one.

As expected, the error is generally increasing with the number of projections, and is comparable with the procedure proposed in [34], up to about $0.8 \%$ after 12 steps. When using coarser meshes, the level of error is obviously higher due to a lower capability of the mesh to represent the function $f$. While refinement (from coarse to fine) is always better than de-refinement (from fine to coarse), transferring from a coarse mesh to a coarse mesh leads to the lower accuracy: this is encouraging for the transfer operation that did not add much additional discrepancy when dealing with different mesh sizes.

\section{Numerical example: thermo-viscoelastic cou- pling}

\subsection{Problem settings}

This example concerns the simulation of a test for identification of a nonlinear thermo-viscoelastic polymeric material subjected to cyclic loading. The temperature is an important parameter, because in some regimes, the Young modulus $E$ of this material exhibits a drop of several orders of magnitude for a temperature increase that can arise from mechanical dissipation during loading, especially after the glass-transition temperature, due to phase transformation $[26,12]$.

For such cases, when the temperature is $\tau=T_{0}+\theta$, with small variations from reference temperature $T_{0}$, and with small perturbations, the state of the material is described at each point of the structure with the following parameters (for a Kelvin-Voigt model type, the stress is partitioned into a reversible and a 
nonrevertible part $\left.\boldsymbol{\sigma}=\boldsymbol{\sigma}^{r}+\boldsymbol{\sigma}^{i}\right)$ :

- temperature $\theta$ associated to entropy $s$,

- strain $\varepsilon$ associated to revertible stress $\boldsymbol{\sigma}^{r}$.

With the formalism of thermodynamics (for nonrevertible processes and internal variable description of the local behavior of the material) the model considered herein, with $\rho$ as specific mass, is given by:

- the free energy $\rho \psi(\theta, \varepsilon)=-\frac{1}{2} \frac{\rho c}{T_{0}} \theta^{2}+\frac{1}{2} \operatorname{Tr}[\varepsilon \boldsymbol{D} \varepsilon]$,

- the dissipation potential $\phi(\underline{Z}, \dot{\varepsilon})=\frac{1}{2} \eta \operatorname{Tr}\left[\dot{\varepsilon}^{2}\right]+\frac{1}{2} \frac{k}{T_{0}} \underline{Z}^{2}$.

$\boldsymbol{D}$ is Hooke operator; it depends on two coefficient for isotropic behavior: the Young modulus $E$ and the Poisson coefficient $\nu$.

For simplicity, the thermal dilatancy is neglected and only the Young modulus is subjected to variations due to the temperature. Moreover we suppose, in a suited temperature range, that this evolution is linear, i.e. $E(\theta)=E_{0}+\theta E_{1}$. In such case, the Hooke operator is also linear with respect to temperature: $\boldsymbol{D}=\boldsymbol{D}_{0}+\theta \boldsymbol{D}_{1}$. $c$ is the specific heat, $\underline{Z}$ is the gradient of temperature, $\eta$ is the viscosity parameter and $k$ is the conductivity coefficient. Finally, $q$ will denote the thermal flux.

The state laws are therefore:

$$
\boldsymbol{\sigma}^{r}=\rho \frac{\partial \psi}{\partial \boldsymbol{\varepsilon}}=\boldsymbol{D}(\theta) \boldsymbol{\varepsilon} \quad \text { and } \quad \rho s=-\rho \frac{\partial \psi}{\partial \theta}=\frac{\rho c}{T_{0}} \theta-\frac{1}{2} \operatorname{Tr}\left[\varepsilon \boldsymbol{D}_{1} \varepsilon\right]
$$

The evolution laws are:

$$
\boldsymbol{\sigma}^{i}=\frac{\partial \phi}{\partial \dot{\varepsilon}}=\eta \dot{\varepsilon} \quad \text { and } \quad \frac{\underline{q}}{T_{0}}=-\frac{\partial \phi}{\partial(\underline{Z})}=-\frac{k}{T_{0}} \underline{Z}
$$

Once the local coupled constitutive behavior has been defined, the global admissibility conditions on the whole domain $\Omega$ must be set; this is done in the next paragraphs.

Concerning the structure-related admissibility, one must have a compatible strain field: $\varepsilon=(\operatorname{grad} \underline{U})_{\mathrm{sym}}$, where the displacement field $\underline{U}$ is regular (it belongs to the corresponding space $\mathcal{U}$ ) and equals the prescribed displacement $\underline{U}_{d}$ on a first part $\partial_{1} \Omega$ of the boundary $\partial \Omega$ of the domain. For momentum conservation, the stress field should also balance the external prescribed forces $\underline{F}_{d}$ on the complementary part of the domain $\partial_{2} \Omega$ (for sake of simplicity, no body force is considered herein): $\boldsymbol{\sigma} \underline{n}_{\mid \partial_{2} \Omega}=\underline{F}_{d}$ and $\underline{\operatorname{div}} \boldsymbol{\sigma}=0$ in $\Omega$. With the constitutive relations, the displacement-oriented variational formulation is, at each time step:

$$
\forall \underline{U}^{\star} \in \mathcal{U}_{0}, \quad \int_{\Omega} \operatorname{Tr}\left[\{\boldsymbol{D}(\theta) \varepsilon(\underline{U})+\eta \varepsilon(\underline{\dot{U}})\} \varepsilon\left(\underline{U}^{\star}\right)\right] d \Omega=\int_{\partial_{2} \Omega} \underline{F}_{d} \cdot \underline{U}^{\star} d S
$$

with $\underline{U} \in \mathcal{U}, \underline{U}_{\mid \partial_{1} \Omega}=\underline{U}_{d}$ and $\mathcal{U}_{0}$ is the subset of $\mathcal{U}$ with null values on $\partial_{1} \Omega$.

Concerning the thermal problem, one must have a compatible temperature gradient: $\underline{Z}=\operatorname{grad} \theta$, where the temperature field $\theta$ is regular (it belongs to 
the corresponding space $\mathcal{T}$ ) and equals the prescribed values $\theta_{d}$ on an other part of the boundary $\partial_{3} \Omega$. The thermal flux must equals the prescribed values on an other part: $q \cdot \underline{n}_{\mid \partial_{4} \Omega}=G_{d}$, the convection condition on the remaining part: $\left.\underline{q} \cdot \underline{n}_{\mid \partial_{5} \Omega}=h \overline{(\theta}-\theta_{\infty}\right)$ ( $h$ is the equivalent convection coefficient, and $\theta_{\infty}$ the corresponding far temperature), as well as the energy balance of the first thermodynamic principle: $\rho \dot{e}+\operatorname{div} q=r+\operatorname{Tr}[\boldsymbol{\sigma} \dot{\varepsilon}] . \quad e=\psi+\tau s$ is the specific internal energy, and $r$ is a thermal source. Following [22] for instance, one gets the local thermal balance equation on $\Omega$ :

$$
-\rho c \dot{\theta}+T_{0} \operatorname{Tr}\left[\varepsilon \boldsymbol{D}_{1} \dot{\varepsilon}\right]=\operatorname{div} \underline{q}-r-\eta \operatorname{Tr}\left[\dot{\varepsilon}^{2}\right]
$$

The corresponding variational formulation is, at each time step:

$$
\begin{aligned}
& \forall \theta^{\star} \in \mathcal{T}_{0}, \quad \int_{\Omega}(\left.\theta^{\star} \rho c \dot{\theta}+\underline{\operatorname{grad}} \theta^{\star} \cdot k \underline{\operatorname{grad}} \theta\right) d \Omega+\int_{\partial_{5} \Omega} \theta^{\star} h \theta d S= \\
& \int_{\Omega} r \theta^{\star} d \Omega-\int_{\partial_{4} \Omega} G_{d} \theta^{\star} d S+\int_{\partial_{5} \Omega} \theta^{\star} h \theta_{\infty} d S+ \\
& \int_{\Omega} \theta^{\star}\left(T_{0} \operatorname{Tr}\left[\varepsilon \boldsymbol{D}_{1} \dot{\varepsilon}\right]+\eta \operatorname{Tr}\left[\dot{\varepsilon}^{2}\right]\right) d \Omega
\end{aligned}
$$

with $\theta \in \mathcal{T}, \theta_{\mid \partial_{3} \Omega}=\theta_{d}$ and $\mathcal{T}_{0}$ is the subset of $\mathcal{T}$ with null values on $\partial_{3} \Omega$.

With such formulation, the last two admissibility equations (9) and (10) are coupled.

For the considered test case, the specimen is a part of a polymethyl methacrylate (PMMA) polymeric plate. So, it as been modeled as a $2 \mathrm{D}$ structure with plane stress assumption, and a constant temperature throughout the thickness. In this case, the convection on each side of the specimen has to be modeled as a source term $r=-\frac{2 h}{e}\left(\theta-\theta_{\infty}\right)$ where $e$ is the thickness of the specimen.

Moreover, we propose to solve the previous coupled equations with a fixed point approach that leads to iterative resolutions of two uncoupled global equations at each time step:

$$
\begin{aligned}
& \forall \underline{U}^{\star} \in \mathcal{U}_{0}, \quad \int_{\Omega} \operatorname{Tr}\left[\left\{\boldsymbol{D}_{0} \varepsilon(\underline{U})+\eta \varepsilon(\underline{\dot{U}})\right\} \varepsilon\left(\underline{U}^{\star}\right)\right] d \Omega=\int_{\partial_{2} \Omega} \underline{F}_{d} \cdot \underline{U}^{\star} d S \\
& -\int_{\Omega} \theta^{p} \operatorname{Tr}\left[\boldsymbol{\varepsilon}^{p} \boldsymbol{D}_{1} \boldsymbol{\varepsilon}\left(\underline{U}^{\star}\right)\right] d \Omega \\
& \forall \theta^{\star} \in \mathcal{T}_{0}, \quad \int_{\Omega}\left(\theta^{\star} \rho c \dot{\theta}+\underline{\operatorname{grad}} \theta^{\star} \cdot k \underline{\operatorname{grad}} \theta+\theta^{\star} \frac{2 h}{e} \theta\right) d \Omega+\int_{\partial_{5} \Omega} \theta^{\star} h \theta d S= \\
& \int_{\Omega} \theta^{\star} \frac{2 h}{e} \theta_{\infty} d \Omega-\int_{\partial_{4} \Omega} G_{d} \theta^{\star} d S+\int_{\partial_{5} \Omega} \theta^{\star} h \theta_{\infty} d S+ \\
& \int_{\Omega} \theta^{\star}\left(T_{0} \operatorname{Tr}\left[\varepsilon^{p} \boldsymbol{D}_{1} \dot{\boldsymbol{\varepsilon}}^{p}\right]+\eta \operatorname{Tr}\left[\left(\dot{\boldsymbol{\varepsilon}}^{p}\right)^{2}\right]\right) d \Omega
\end{aligned}
$$

$\theta^{p}, \varepsilon^{p}$ and $\dot{\varepsilon}^{p}$ are predictors for the temperature, strain and strain rate. They will be precised in the next Sections. 


\subsection{Feasibility of the approach}

For this first test case, the specimen of Figure 8 is considered.

\subsubsection{Spatial discretization}

The domain $\Omega$ is discretized independently for the structure with a mesh $\Omega_{s}$ and for the thermal problem with a second mesh $\Omega_{t}$. Thanks to the symmetries of the problem, only one fourth of the specimen has been meshed, see Figure 8 (the height of the meshed part is $L=5 \mathrm{~cm}$, the minimum width is $2 \mathrm{~cm}$, the maximum width, $3.5 \mathrm{~cm}$, and the radius, $5 \mathrm{~cm}$ ), independently for the viscoelastic problem and the thermal problem. These meshes are fully incompatible; the mesh used for the viscoelastic problem contains 171 6-node-triangle elements (with 3 Gauss points), while the mesh for the thermal problem contains 149 3-node-triangle elements (with 3 Gauss points for computing the conduction matrix). The prescribed displacement is a sine with a period $T / 3=3 \mathrm{~s}$ and with a maximum value $u_{0}=0.25 \mathrm{~mm}$. The initial relative temperature is $\theta_{0}=\theta_{d}=\theta_{\infty}=-7 \mathrm{~K}$ (distance to the reference temperature $T_{0}$ which is the glass-transition temperature). The characteristics of the material are given in Table $2[12,26]$.
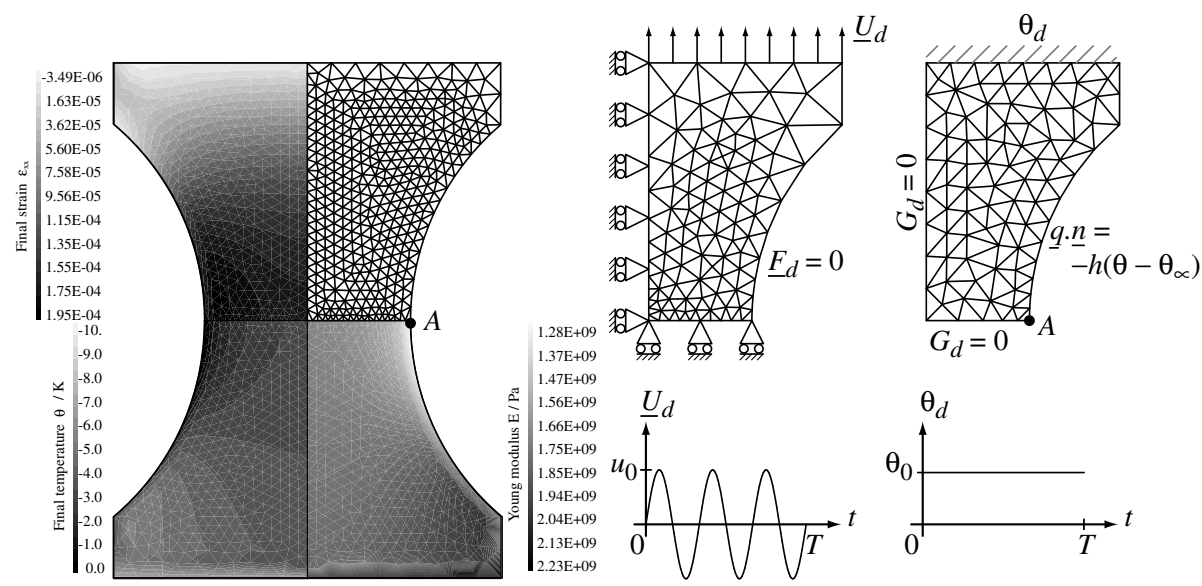

Figure 8: Test case problem. Left: specimen geometry and reference mesh of one fourth of the domain; center: viscoelastic mesh; right: thermic mesh

The previous decoupled problems are now expressed in terms of vectors of temperature unknowns $\boldsymbol{\theta}$ and displacement unknowns $\boldsymbol{u}$ :

$$
\begin{gathered}
\boldsymbol{K}_{0} \boldsymbol{u}+\boldsymbol{N} \dot{\boldsymbol{u}}=\boldsymbol{f}_{d}-\boldsymbol{b}_{\boldsymbol{\sigma}}^{T} \boldsymbol{\sigma}^{p} \\
\boldsymbol{C} \dot{\boldsymbol{\theta}}+(\boldsymbol{K}+\boldsymbol{H}) \boldsymbol{\theta}=\boldsymbol{g}_{d}+\boldsymbol{b}_{q}^{T} \boldsymbol{q}^{p}
\end{gathered}
$$

$\boldsymbol{f}_{d}$ are the external generalized forces for the structural problem, $\boldsymbol{g}_{d}$ are the external generalized fluxes for the thermal problem (both contain the prescribed right hand sides). $\boldsymbol{\sigma}^{p}$ and $\boldsymbol{q}^{p}$ are the following coupling terms:

$$
\boldsymbol{\sigma}^{p}=\left(\mathcal{P}_{s t} \boldsymbol{b}_{\theta} \theta^{p}\right) \cdot \boldsymbol{D}_{1}\left(\boldsymbol{b}_{\boldsymbol{\varepsilon}} \boldsymbol{u}^{p}\right)
$$




\begin{tabular}{ll}
\hline initial Young modulus & $E_{0}=1325 \mathrm{MPa}$ \\
sensitivity of Young modulus v.r. to temp. & $E_{1}=-130 \mathrm{MPa} \cdot \mathrm{K}^{-1}$ \\
Poisson coefficient & $\nu=0.4$ \\
viscosity parameter & $\eta=10^{10} \mathrm{~Pa} \cdot \mathrm{s}$ \\
\hline specific heat & $c=1470 \mathrm{~J} \cdot \mathrm{kg}^{-1} \cdot \mathrm{K}^{-1}$ \\
conductivity coefficient & $k=0.18 \mathrm{~W} \cdot \mathrm{m}^{-1} \cdot \mathrm{K}^{-1}$ \\
specific mass & $\rho=1190 \mathrm{~kg} \cdot \mathrm{m}^{-3}$ \\
\hline reference temperature & $T_{0}=385 \mathrm{~K}$ \\
equivalent convection coefficient & $h=25 \mathrm{~J} \cdot \mathrm{m}^{-2} \cdot \mathrm{K}^{-1}$ \\
far temperature & $\theta_{\infty}=-7 \mathrm{~K}$ \\
specimen thickness & $e=2 \mathrm{~mm}$ \\
\hline
\end{tabular}

Table 2: Material characteristics for a PMMA polymer, and other parameters

$$
\boldsymbol{q}^{p}=\mathcal{P}_{t s}\left[\left(\boldsymbol{b}_{\varepsilon} \dot{\boldsymbol{u}}^{p}\right):\left(T_{0} \boldsymbol{D}_{1} \boldsymbol{b}_{\boldsymbol{\varepsilon}} \boldsymbol{u}^{p}+\eta \boldsymbol{b}_{\varepsilon} \dot{\boldsymbol{u}}^{p}\right)\right]
$$

$\boldsymbol{b}_{\theta}$ is the operator that transfers the vector of discretized nodal temperature values, to the integration points of $\Omega_{t}$, i.e. with the previous notations, $\boldsymbol{b}_{\theta}=\mathcal{N}_{t}$. $\boldsymbol{b}_{q}=\boldsymbol{W}_{t} \mathcal{N}_{t}$ is the other operator associated to the "mass" matrix on $\Omega_{t}$ (or equivalently associated to the conduction matrix). The equivalent operators associated to the rigidity matrix are $\boldsymbol{b}_{\boldsymbol{\varepsilon}}$ (transferring a nodal displacement to the strain at integration points) and $\boldsymbol{b}_{\boldsymbol{\sigma}}=\boldsymbol{W}_{s} \boldsymbol{b}_{\boldsymbol{\varepsilon}}$.

$\mathcal{P}_{s t}$ and $\mathcal{P}_{t s}$ are the transfer operators between the structural domain $\Omega_{s}$ and the thermal domain $\Omega_{t}$, at integration points. It can be noticed that, as the structural fields that have to be transferred are related to stress and strain, the underlying mesh used for transferring the fields is not directly $\Omega_{s}$ (with 6-node triangles), but the mesh containing 3-node triangles based only on the vertex of the previous elements. With 3 Gauss points, these elements give invertible elementary mass matrices.

Two products are involved in the right hand sides: the single dot denotes the entry-by-entry product of two vectors, while the double dot denotes the corresponding tensorial product between two tensor fields represented by the vector of their values.

$\boldsymbol{K}_{0}$ is the rigidity matrix obtained with the first part of Hooke operator $\boldsymbol{D}_{0} . \boldsymbol{N}$ is the viscosity matrix; it can be numerically computed with the same routine as for the rigidity, provided with a null Poisson coefficient, and $\eta$ as Young modulus. $\boldsymbol{C}$ is the conduction matrix; it is similar to the mass matrix with $\rho c$ as specific mass. Finally, $\boldsymbol{K}$ is the conductivity matrix and $\boldsymbol{H}$ is the total convection matrix.

\subsubsection{Time discretization}

Two different time discretizations could also be chosen for each physics (structural and thermal), as soon as adequate time transfer operator are defined, but this is actually not under the scope of this article. Therefore we will consider only one time discretization, and the integration scheme is chosen to be the $\theta$-method (with $\theta=1$ : implicit Euler method, unconditionally stable).

The integration of a variable $X$ given at each time step $t_{i}$ with its value $X_{i}$ is then: $X_{i+1}=X_{i}+\left(t_{i+1}-t_{i}\right) \dot{X}_{i+\theta}$ (with the notation $\left.\dot{X}_{i+\theta}=\theta \dot{X}_{i+1}+(1-\theta) \dot{X}_{i}\right)$ 
and the associated derivation scheme is:

$$
\dot{X}_{i+1}=\frac{1}{\theta\left(t_{i+1}-t_{i}\right)}\left(X_{i+1}-X_{i}\right)-\frac{1-\theta}{\theta} \dot{X}_{i}
$$

In the following, we will only consider the case where the time step is constant $t_{i+1}-t_{i}=\Delta t$. The initial problem is transformed into a step-by-step incremental problem; at time $t_{i+1}$, it reads:

$$
\begin{gathered}
\left(\boldsymbol{K}_{0}+\frac{1}{\theta \Delta t} \boldsymbol{N}\right) \boldsymbol{u}_{i+1}=\boldsymbol{N}\left(\frac{1}{\theta \Delta t} \boldsymbol{u}_{i}+\frac{1-\theta}{\theta} \dot{\boldsymbol{u}}_{i}\right)+\boldsymbol{f}_{d i+1}-\boldsymbol{b}_{\boldsymbol{\sigma}}^{T} \boldsymbol{\sigma}^{p} \\
\left(\frac{1}{\theta \Delta t} \boldsymbol{C}+\boldsymbol{K}+\boldsymbol{H}\right) \boldsymbol{\theta}_{i+1}=\boldsymbol{C}\left(\frac{1}{\theta \Delta t} \boldsymbol{\theta}_{i}+\frac{1-\theta}{\theta} \dot{\boldsymbol{\theta}}_{i}\right)+\boldsymbol{g}_{d i+1}+\boldsymbol{b}_{q}^{T} \boldsymbol{q}^{p}
\end{gathered}
$$

\subsubsection{Iterative solution algorithm}

For a given time step $t_{i+1}$, a fixed point method consists of selecting a predictor, and then to iterate as long as necessary for consistency and precision to recover the fully coupled solution. Structural problem and thermal problems are uncoupled at each iteration of the fixed point method. The predictors can be selected as: $\boldsymbol{u}^{p}=\boldsymbol{u}_{i}+\Delta t \dot{\boldsymbol{u}}_{i}, \dot{\boldsymbol{u}}^{p}=\dot{\boldsymbol{u}}_{i}, \boldsymbol{\theta}^{p}=\boldsymbol{\theta}_{i}+\Delta t \dot{\boldsymbol{\theta}}_{i}$, which are all explicit at time step $t_{i+1}$.

Once these predictors have been set up, the fixed point consists of repeating the sequence for the same time step: (i) solve previous equations to get $\boldsymbol{u}_{i+1}$ and $\boldsymbol{\theta}_{i+1}$, (ii) use integration scheme to get $\dot{\boldsymbol{u}}_{i+1}$ and $\dot{\boldsymbol{\theta}}_{i+1}$, (iii) update the predictors with these values. The fixed point can be stopped by monitoring the residual in equilibrium with the current approximate solution. Figure 9 illustrates several variants of fixed-point-like methods. For staggered schemes, the step 1 consists of using value and predictor to advance one physics; the step 2 uses the new computed value as predictor and advances the second physics; the step 3 and over are eventual correction cycles. For parallel scheme, the step 1 uses values and predictors to advance in parallel the two physics; step 2 is eventual cyclings for correction (fixed point). Other decoupled iterative solution scheme can be developed such as those in $[25,13,16]$ for instance.
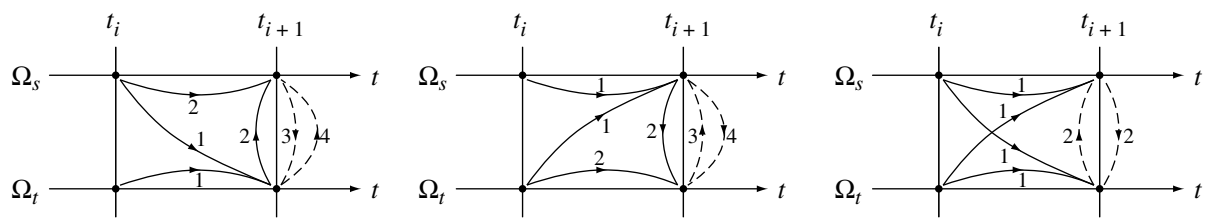

Figure 9: Partitioning schemes. Left and center: staggered (Gauss-Seidel-like); right: parallel (Jacobi-like).

With the chosen splitting and use of predictors, and with a constant time step, one can remark that the left hand side matrices are always constant, and therefore only need to be factorized once. 
During these iterations, the different fields have to be exchanged between structural problem and thermal problem in order to compute the coupling terms in the right hand sides. This is done with the transfer operators $\mathcal{P}_{s t}$ and $\mathcal{P}_{t s}$.

For the considered test case, the number of time steps has been chosen to be 120 and the iterative scheme is the parallel one. The stopping criteria for the fixed point method is to get a relative energy norm error of both viscoelastic and thermal equilibrium residuals less than $10^{-6}$. The number of iterations has been observed to be no more than 4 for any time step, and is often only 3 . Figure 10 reports the evolution of the maximum and minimum temperatures. To compare with a reference solution, the same problem has been solved with the reference compatible mesh of Figure 8-left that contains 746 6-node-triangle elements. The interpolation is $\mathrm{P} 2$ for the displacement and P1 for the temperature, both continuous throughout the element edges (with 3 Gauss points per element) [7]. The non-trivial evolution of temperature is due to the material model and to structural effects. To have a comparison point, the case of an homogeneous solution for an adiabatic problem $(q=0, r=0)$ submitted to a prescribed strain rate $\left(\dot{\varepsilon}(t)=\dot{\varepsilon}_{0} \cos (\omega t / 2)\right.$ with $\dot{\varepsilon}_{0}=\omega u_{0} / 2 L$ and $\left.\omega=12 \pi / T\right)$ can be found analytically:

$$
\frac{\theta^{0}(t)-\theta^{0}(0)}{T_{0}}=\frac{1}{2} \frac{\dot{\varepsilon}_{0}^{2}}{\rho c \omega}\left[\frac{2 E_{1}}{\omega}(1-\cos \omega t)+\frac{\eta}{T_{0}}(\omega t+\sin \omega t)\right]
$$

Its evolution is also plotted in Figure 10. The finite element code Cast3M ${ }^{\mathrm{TM}}$ (CEA Saclay, France) has been used for pre-processing, while the iterative method to solve the coupled thermo-viscoelastic problem has been implemented within Matlab ${ }^{\mathrm{TM}}$ environment.

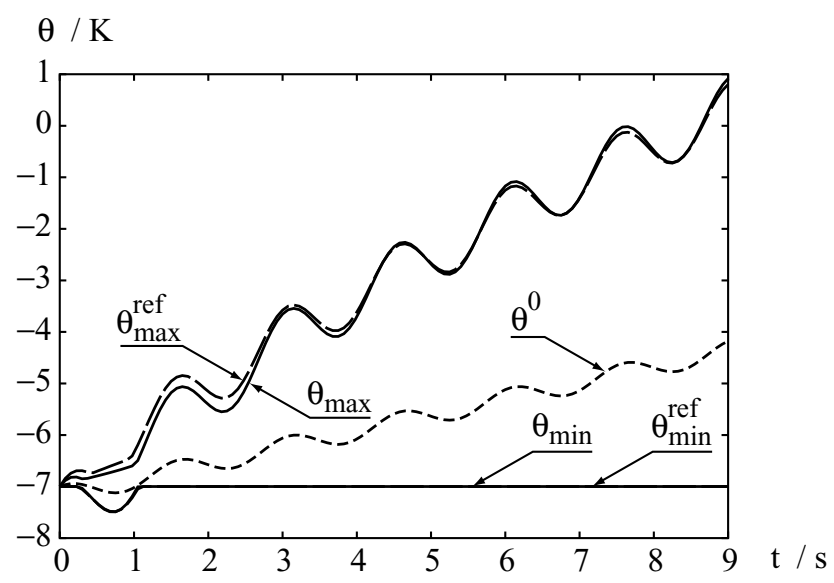

Figure 10: Maximal temperature evolutions for reference problem $\left(\theta^{\text {ref }}\right)$, nonmatching mesh problem $(\theta)$, and homogeneous adiabatic problem $\left(\theta^{0}\right)$

The proposed mortar-like approach gives satisfactory results when compared to a fine meshed solution. 


\subsection{Comparison with classical transfer scheme}

In order to compare the proposed approach with the classical collocation transfer method, this last one should be adapted to the case of sample fields: let us consider a sample field $\mathcal{E}_{1}$; the collocation transfer consists of:

- element by element extrapolation $\boldsymbol{E}_{1}=\mathcal{L}_{1} \mathcal{E}_{1}$

- interpolation to the target integration points $\mathcal{E}_{2}=\boldsymbol{N}_{1}\left(x_{2}\right) \boldsymbol{E}_{1}$, where $x_{2}$ are the natural coordinates of the integration points of mesh $\Omega_{2}$ in elements of $\Omega_{1}$.

The overall procedure is $\mathcal{E}_{2}=\mathcal{P}_{21}^{c} \mathcal{E}_{1}$.

A test case with higher gradients in the solution is proposed on Figure 11. The problem is similar as the previous one, but with a plate with a hole in its center as a specimen (the height of the meshed part is $L=5 \mathrm{~cm}$, the width is $3.5 \mathrm{~cm}$, and the radius, $2 \mathrm{~cm}$ ). Material characteristics, loadings, time discretization, convection conditions, iterative algorithm... are the same as in the previous Section.

Figure 12 reports the evolution of the temperature $\theta$ on the horizontal symmetry axis of the whole specimen, at point $B$ of Figure 11, located on the hole. Three different simulations are reported: the reference solution is obtained with the unique fine mesh of Figure 11; with the two different meshes of the same Figure, the 'mortar' and the 'collocation' methods are used to get the other simulations. The same results are also plotted for the first test case, this time at point $A$ of Figure 8 , for which the three simulations give very similar results.
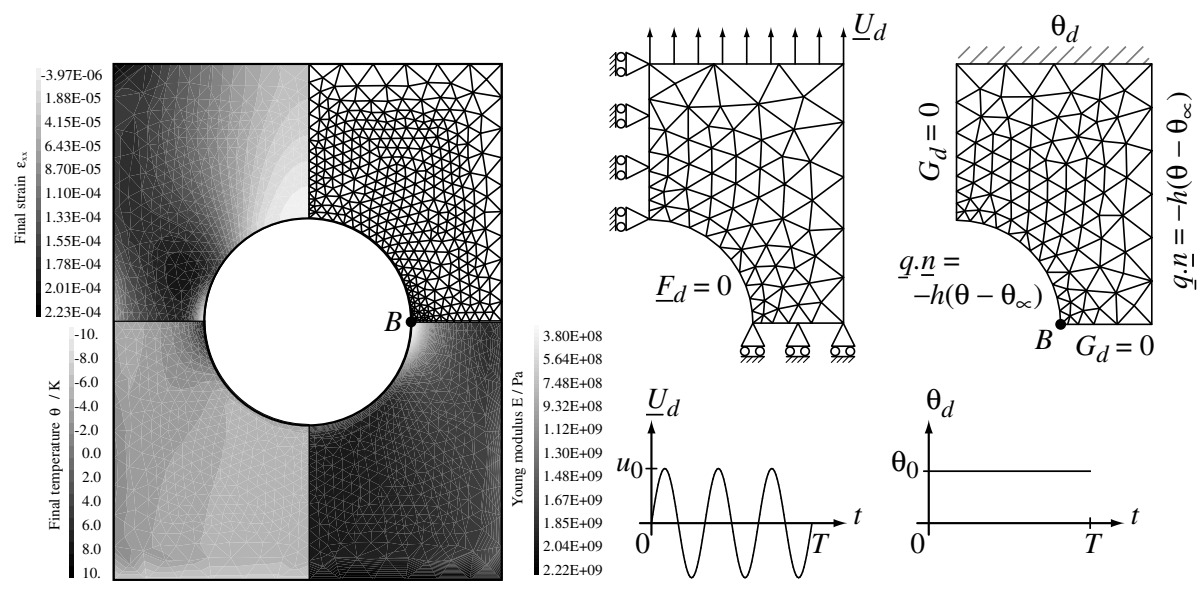

Figure 11: Second test case problem. Left: specimen geometry and reference mesh of one fourth of the domain; center: viscoelastic mesh; right: thermic mesh.

Clearly, the proposed mortar-like approach leads to significant improvement when compared to a standard collocation transfer method. 


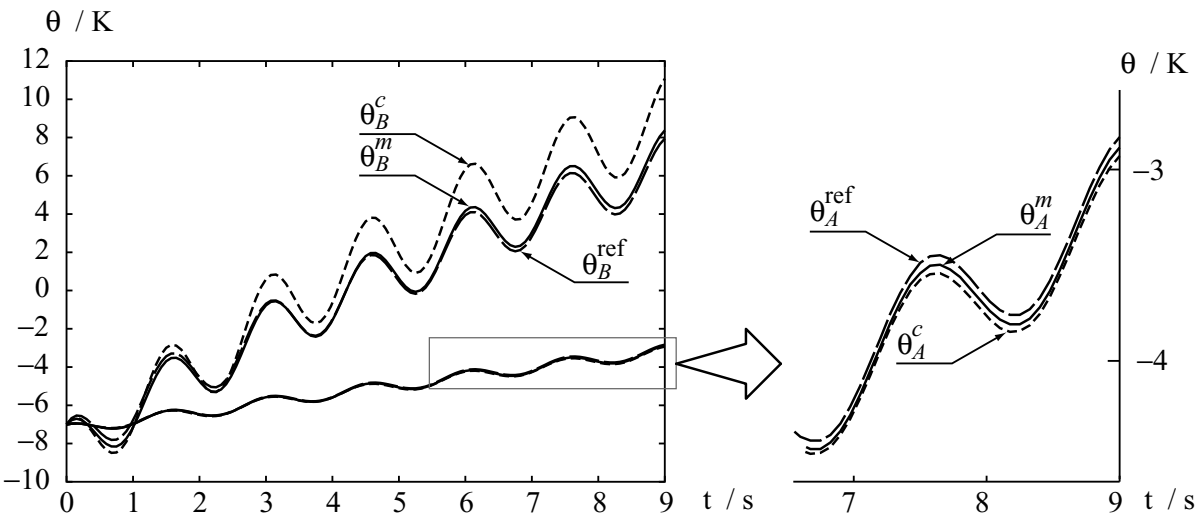

Figure 12: Temperature evolutions for reference problem $\left(\theta^{\text {ref }}\right)$, nonmatching mesh problem with 'mortar' approach $\left(\theta^{m}\right)$, and 'collocation' approach $\left(\theta^{c}\right) ; A$ and $B$ refer to the first and second test cases

\subsection{Test case with jumps in the solution}

\subsubsection{Non smoothness and projection}

The proposed approach does not involve a smoothing of the field to be transferred. As noticed in [29], the internal variable fields are not involved in spatial derivative in the general variational formulation; this allows the variational enforcement of continuity to be imposed element by element. One can expect a smoothing to decrease the gradient in the solution, especially if this solution is not continuous (see the laminate example in the following). Results obtained in [29] indicates effectively that the quality assessment of the solution is better without smoothing (the reader can find in this article a comparison between a variationally consistent transfer, a weak enforcement, and a smoothing transfer). As mentioned also in [34], smoothing can lead to lack of self-consistency. Nevertheless, an other requirement is to limit eventual spurious local extrema while projecting.

Concerning such spurious extrema in the projected field, due to the extrapolation and the nonsmooth field descriptions (potential jump throughout the element edges), a simple test can illustrate the behavior of the proposed projection operators: let us consider the $1 \mathrm{D}$ case of a field with a jump at the origin: $e_{1}(x)=\operatorname{sgn}(x)$, exactly represented on a one dimensional mesh $\Omega_{1}$ constituted with 2-node segments, each possessing 2 gaussian integration points. Let us now consider a mesh $\Omega_{2}$ with the same elements, but with an element spanning from $x=-h_{2} / 2$ to $x=h_{2} / 2$. The coordinates of the target gaussian integration points on this last element are: $x_{2}= \pm \frac{h_{2}}{2 \sqrt{3}}[42]$. Obviously, the projected field $e_{2}$ on $\Omega_{2}$ is linear on each element before being interpolated on the target integration points; for the considered test, its standard average is null, so: $e_{2}(x)=\beta x$. To get its values at integration points of the central element of mesh $\Omega_{2}$, let us consider the generalized average of both fields, with a linear test function $\varphi(x)=x$.

Extrapolation on $\Omega_{1}$ still maintains the sign function values. On the central 
element of $\Omega_{2}$,

$$
\int_{-h_{2} / 2}^{h_{2} / 2} \varphi(x) \operatorname{sgn}(x) d x_{1}=h_{2}^{2} / 4 \quad \text { and } \quad \int_{-h_{2} / 2}^{h_{2} / 2} \varphi(x) \beta x d x_{2}=\frac{2}{3} \beta h_{2}^{3} / 8
$$

Equalizing the previous values lead to $\beta=3 / h_{2}$. Finally, the values at Gauss points of the central element of $\Omega_{2}$ are $\beta x_{2}= \pm \sqrt{3} / 2 \approx \pm 0.87$, see Figure 13 .

Therefore, the values are independent of the mesh size $h_{2}$, and no oscillation occurs when refining $\Omega_{2}$.
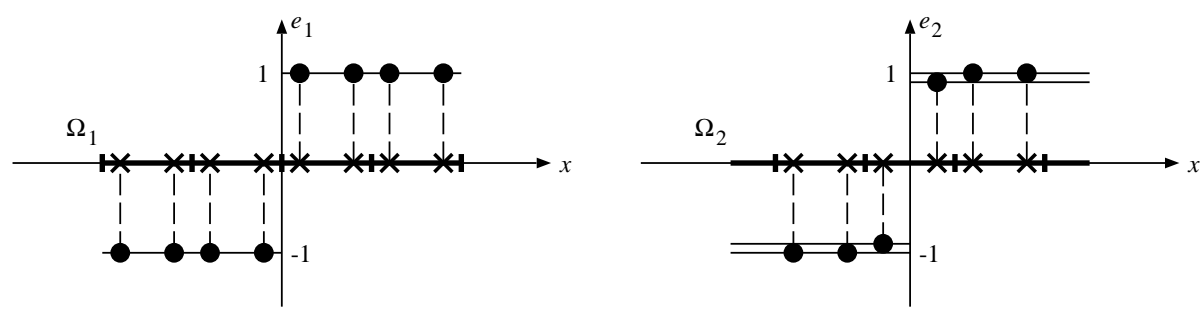

Figure 13: Illustration of a non-smooth field projection

\subsubsection{A laminate simulation}

To test the transfer procedure on a problem that exhibits jumps in the solution, the heterogeneous problem of a laminate is proposed. Such a structure is made with a stack of alternate steel and polymer plates (here, we use again PMMA). We are interested in the stress field at the junctions of the materials to check for delamination, while the temperature field do not require to be computed up to a high accuracy. We therefore need for a two scale modeling for this problem. This is achieved by choosing a fine mesh for the stress estimation, and a rough mesh for the thermal problem. Figure 14 shows the meshes used (the coarsest ones of a whole set) for one fourth of the structure with the corresponding symmetry boundary conditions (the width of the meshed part is $4 \mathrm{~cm}$, the steel plates are $2 \mathrm{~mm}$ thick, and the polymer plates, $5 \mathrm{~mm}$ ).

In this test, the chosen thermal mesh do not match the material separation surfaces. The thermal model therefore requires thermal material coefficients derived from the microstructure description (the stratification). Here, we simply use a mixture law (the volume ratio of steel is about $28 \%$ ). The material coefficients for the steel are given in Table 3.

A similar problem occurs with the definition of a reference solution, since the initial problem is modeled with different scales for the two physics involved. The consistent reference solution is therefore obtained with identical fine meshes for the two physics, but the temperature field if then transferred to the coarse mesh in order to be 'homogenized'. This hybrid reference solution is thus the viscoelastic quantities, as computed of the fine mesh, and the projected thermal quantities on the coarse mesh.

Figure 15 shows the evolutions of maximum and minimum temperatures. On the left of this Figure, the reference solution, as well as its projection with either collocation or mortar projector. Both are quite similar and close to the reference. 

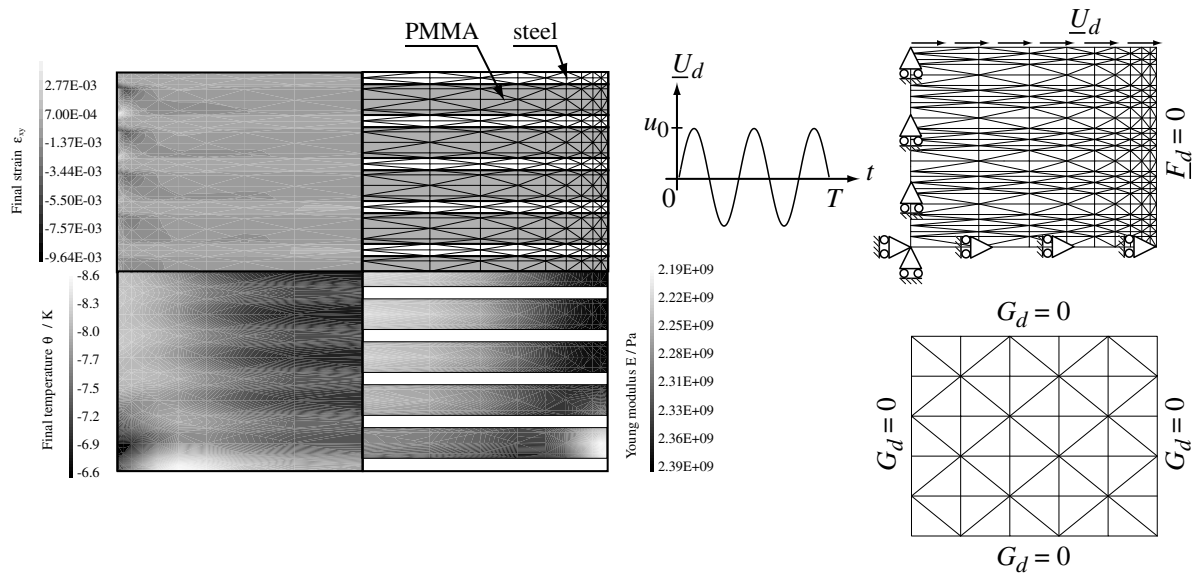

Figure 14: Steel-PMMA laminate with the final solution fields (left) and coarsest tested meshes (right)

\begin{tabular}{ll}
\hline initial Young modulus & $E_{0}^{\prime}=200 \mathrm{GPa}$ \\
sensitivity of Young modulus v.r. to temp. & $E_{1}^{\prime}=0$ \\
Poisson coefficient & $\nu^{\prime}=0.33$ \\
viscosity parameter & $\eta^{\prime}=0$ \\
\hline specific heat & $c^{\prime}=438 \mathrm{~J} \cdot \mathrm{kg}^{-1} \cdot \mathrm{K}^{-1}$ \\
conductivity coefficient & $k^{\prime}=20 \mathrm{~W} \cdot \mathrm{m}^{-1} \cdot \mathrm{K}^{-1}$ \\
specific mass & $\rho^{\prime}=7800 \mathrm{~kg} \cdot \mathrm{m}^{-3}$ \\
\hline reference temperature & $T_{0}=385 \mathrm{~K}$ \\
equivalent convection coefficient & $h=0$ \\
\hline
\end{tabular}

Table 3: Material characteristics for the steel in the bimaterial laminate test case.

Therefore, one of them (the mortar one) is used as a projected reference to compare the simulations performed with different meshes on the right of the Figure. This time, with exchanges of information in both directions all along the simulation, a neat difference occurs between the mortar and the collocation approach: the proposed approach is clearly closer to the reference, even for this nonsmooth problem.

To test the behavior of the proposed approach when changing the discretizations, the laminate test is treated with successive finer meshes for both the viscoelastic and the thermic finite element models. The previous comparison was made with the coarsest meshes for the two physics. The viscoelastic mesh is always the most refined one and is selected for computing a reference solution (therefore, the reference changes as soon as the viscoelastic mesh is changed). This reference is no more projected in the following. To measure the differences obtained with mortar or collocation approaches, errors on the evolution on $[0, T]$ 


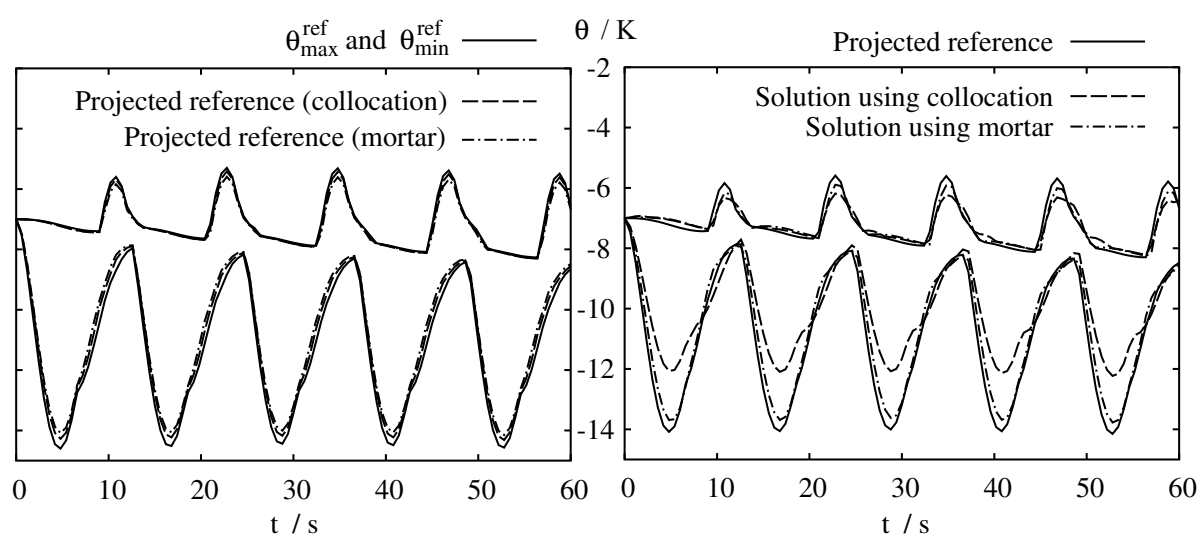

Figure 15: Evolutions of maximum and minimum temperatures for steel-PMMA laminate, for reference and projected reference simulations (left), for mortar and collocation approaches (right)

\begin{tabular}{ccccccc}
\hline$n_{\text {ref }}=n_{v}$ & $n_{t}$ & $e_{\max } \%$ & $e_{\max } \%$ & $e_{\min } \%$ & $e_{\min } \%$ & $\frac{e_{\min } \text { collocation }}{n}$ \\
& & mortar & collocation & mortar & collocation & \\
\hline 504 & 50 & 4.799 & 5.603 & 6.636 & 13.116 & 1.98 \\
\hline 1176 & 50 & 6.268 & 7.032 & 7.464 & 13.883 & 1.86 \\
1176 & 200 & 1.298 & 4.290 & 1.269 & 5.231 & 4.12 \\
\hline 4074 & 50 & 6.113 & 6.828 & 7.346 & 13.696 & 1.86 \\
4074 & 200 & 1.198 & 4.338 & 1.254 & 5.253 & 4.19 \\
4074 & 800 & 0.702 & 0.736 & 0.736 & 2.360 & 3.21 \\
\hline
\end{tabular}

Table 4: Influence of the discretization: results with different references

of the minimum and maximum temperature, $\theta_{\min }$ and $\theta_{\max }$, are used:

$$
e_{\max }^{2}=\frac{\int_{0}^{T}\left(\theta_{\max }-\theta_{\max }^{\mathrm{ref}}\right)^{2} \frac{d t}{T}}{\int_{0}^{T}\left(\theta_{\max }^{\mathrm{ref}}\right)^{2} \frac{d t}{T}} \quad \text { and } \quad e_{\min }^{2}=\frac{\int_{0}^{T}\left(\theta_{\min }-\theta_{\min }^{\mathrm{ref}}\right)^{2} \frac{d t}{T}}{\int_{0}^{T}\left(\theta_{\min }^{\mathrm{ref}}\right)^{2} \frac{d t}{T}}
$$

Table 4 reports the values of these errors for the mortar and the collocation approaches, when changing the number of 6-node triangular elements $n_{v}$ of the viscoelastic mesh and the number of 3-node triangular elements $n_{t}$ of the thermic mesh.

As expected, for each choice of viscoelastic discretization, the error decreases when refining the thermic mesh. In each case, the proposed approach leads to a lower error when compared to the collocation method. 


\section{Conclusions}

A field transfer procedure between two incompatible meshes has been proposed. Based on a geometric construction, it is independent of the application. Nevertheless, it features the conservation of duality when transferring fields in both directions between the meshes. It is suited for transferring information stored at integration points and uses only local element-by-element matrices: based on a discontinuous mortar-like approach, it does not require any global mass matrix inversion. Its ability to conserve duality makes it suitable for partitioning iterative solution methods. The feasibility has been illustrated on a strongly coupled thermo-viscoelastic problem. This was a 2D plane stress problem, but the proposed transfer operation is fully applicable for $3 \mathrm{D}$ problems.

The authors wish to thank their colleagues A. Chrysochoos and R. Peyroux for fruitful discussions about thermo-viscoelasticity.

\section{References}

[1] F. B. Belgacem. The mortar finite element method with Lagrange multipliers. Numerische Mathematik, 84(2):173-197, 1999.

[2] F. B. Belgacem and Y. Maday. The mortar finite element method for three dimensional finite elements. Mathematical Modelling and Numerical Analysis, 31:289-302, 1997.

[3] H. Ben Dhia. Multiscale mechanical problems: the Arlequin method. Comptes Rendus de l'Académie des Sciences, Paris, (326):899-904, 1998. Série IIb.

[4] H. Ben Dhia. Numerical modelling of multiscale problems: the Arlequin method. In W. Wunderlich, editor, Proceedings of the European Conference on Computational Mechanics, Munich, 1999.

[5] C. Bernardi, Y. Maday, and A. T. Patera. Domain decomposition by the mortar element method. In H. Kaper et al, editor, Asymptotic and numerical methods for partial differential equations with critical parameters, pages 296-286. Reibdel, Dordrecht, 1993.

[6] C. Bernardi, Y. Maday, and A. T. Patera. A new nonconforming approach to domain decomposition: the mortar element method. In H. Brezzi et al, editor, Nonlinear partial differential equations and their applications, pages 13-51. Paris, 1994.

[7] F. Brezzi and M. Fortin. Mixed and Hybrid Finite Element Methods, volume 15 of Computational Mathematics. Springer, 1991.

[8] X.-C. Cai, M. Dryja, and M. Sarkis. Overlapping non-matching grid mortar element methods for elliptic problems. SIAM Journal of Numerical Analysis, 36:581-606, 1999.

[9] P. J. Davis and P. Rabinowitz. Methods of numerical integration. In Computer Science and Applied Mathematics. Academic Press Inc., Orlando, second edition, 1984. 
[10] D. A. Dunavant. High degree efficient symmetrical Gaussian quadrature rules for the triangle. International Journal for Numerical Methods in Engineering, (21):1129-1148, 1985.

[11] D. Dureisseix, P. Ladevèze, and B. A. Schrefler. A LATIN computational strategy for multiphysics problems: application to poroelasticity. International Journal for Numerical Methods in Engineering, 56:1489-1510, 2003.

[12] G. W. Ehrenstein. Polymeric materials: structure - properties - applications. Hanser Gardner, 2001.

[13] C. Farhat and M. Lesoinne. Two efficient staggered algorithms for the serial and parallel solution of three-dimensional nonlinear transient aeroelastic problems. Computer Methods in Applied Mechanics and Engineering, 182:499-515, 2000.

[14] C. Farhat, M. Lesoinne, and P. Le Tallec. Load and motion transfer algorithms for fluid/structure interaction problems with non-matching discrete interfaces. Computer Methods in Applied Mechanics and Engineering, 157:95-114, 1998.

[15] C. Farhat and D. Rixen. Encyclopedia of Vibration, chapter Linear Algebra, pages 710-720. Academic Press, 2002.

[16] C. A. Felippa, K. C. Park, and C. Farhat. Partitioned analysis of coupled mechanical systems. Computer Methods in Applied Mechanics and Engineering, 190:3247-3270, 2001.

[17] G. Golub and C. Van Loan. Matrix Computations. The Johns Hopkins University Press, Baltimore, third edition, 1996.

[18] J. Grandy. Conservative remapping and region overlays by intersecting arbitrary polyhedra. Journal of Computational Physics, 148:433-466, 1999.

[19] M. W. Heinstein and T. A. Laursen. A three dimensional surface-to-surface projection algorithm for non-coincident domains. Communications in $\mathrm{Nu}$ merical Methods for Engineering, 19:421-432, 2003.

[20] C. Kim, R. D. Lazarov, J. E. Pasciak, and P. S. Vassilevski. Multiplier spaces for the mortar finite element method in three dimensions. SIAM Journal of Numerical Analysis, 39(2):519-538, 2001.

[21] P. Ladevèze and D. Dureisseix. A micro/macro approach for parallel computing of heterogeneous structures. International Journal for Computational Civil and Structural Engineering, (1):18-28, 2000.

[22] J. Lemaitre and J.-L. Chaboche. Mechanics of Solid Materials. Cambridge University Press, 1994.

[23] N. Maman and C. Farhat. Matching fluid and structure meshes for aeroelastic computations: a parallel approach. Computers \& Structures, 54(4):779$785,1995$. 
[24] K. M. Mao and C. T. Sun. A refined global-local finite element analysis method. International Journal for Numerical Methods in Engineering, (32):29-43, 1991.

[25] R. Matteazzi, B. Schrefler, and R. Vitaliani. Comparisons of partitioned solution procedures for transient coupled problems in sequential and parallel processing, pages 351-357. Advances in Computational Structures Technology. Civil-Comp Ltd, 1996.

[26] N. G. McCrum, B. E. Read, and G. Williams. Anelastic and dielectric effects in polymeric solids. Dover Publications, 1991.

[27] A. K. Noor. Global-local methodologies and their application to non-linear analysis. Finite Element Analysis, (2):333-346, 1986.

[28] R. Ohayon and J.-P. Morand. Fluid-Structure Interaction: Applied Numerical Methods. John Wiley \& Sons, 1995.

[29] A. Orlando and D. Perić. Analysis of transfer procedures in elastoplasticity based on the error in the constitutive equations: Theory and numerical illustration. International Journal for Numerical Methods in Engineering, 60(9):1595-1631, 2004.

[30] M. Ortiz and J. J. Quigley. Adaptive mesh refinement in strain localization problems. Computer Methods in Applied Mechanics and Engineering, 90(13):781-804, 1991.

[31] M. Ortiz and L. Stainier. The variational formulation of viscoplastic constitutive updates. Computer Methods in Applied Mechanics and Engineering, 171:419-444, 1999.

[32] D. Perić, C. Hochard, M. Dutko, and D. R. J. Owen. Transfer operators for evolving meshes in small strain elastoplasticity. Computer Methods in Applied Mechanics and Engineering, 137(3-4):331-344, 1996.

[33] D. Perić, M. Vaz Jr., and D. R. J. Owen. On adaptive strategies for large deformation of elasto-plastic solids at finite strains: computational issues and industrial applications. Computer Methods in Applied Mechanics and Engineering, 176:279-312, 1999.

[34] M. M. Rashid. Material state remapping in computational solid mechanics. International Journal for Numerical Methods in Engineering, 55:413-450, 2002.

[35] A. Srikanth and N. Zabaras. An updated lagrangian finite element sensitivity analysis of large deformations using quadrilateral elements. International Journal for Numerical Methods in Engineering, 52:1131-1163, 2001.

[36] J. L. Steger and J. A. Benek. On the use of composite grid schemes in computational aerodynamics. Computer Methods in Applied Mechanics and Engineering, 64:301-320, 1987.

[37] J. L. Steger, F. C. Dougherty, and J. A. Benek. A Chimera grid scheme. In K. N. Ghia and U. Ghia, editors, Advances in Grid Generation, ASME, pages $59-69,1983$. 
[38] P. Villon, H. Borouchaki, and K. Saanouni. Transfert de champs plastiquement admissibles. Comptes Rendus Mécanique, Académie des Sciences, Paris, (220):313-318, 2002.

[39] J. D. Whitcomb. Iterative global/local finite element analysis. Computers \& Structures, (40):1027-1031, 1991.

[40] B. I. Wohlmuth. A mortar finite element method using dual spaces for the Lagrange multipliers. SIAM Journal of Numerical Analysis, 39:989-1012, 2000 .

[41] O. C. Zienkiewicz and R. L. Taylor. The finite element patch test revisited a computer test for convergence, validation and error estimates. Computer Methods in Applied Mechanics and Engineering, 149:223-254, 1997.

[42] O. C. Zienkiewicz and R. L. Taylor. The finite element method. Butterworth Heinemann, fifth edition, 2000.

\section{A Special case of under-integration}

Some difficulties arise as soon as the elementary mass matrices are singular. This is the case when the number of integration points is smaller than the number of nodes. Such a situation happens for instance when, in structural analysis, the set of Gauss points for evaluation of the stiffness matrix (and where the stress field is usually defined) is also used for computing the mass matrix, since polynomials to be integrated for the stiffness have a lower degree than those for the mass. As seen before, the interpolation operator is then no more injective, and the element mass matrices are singular. This requires modifications of the extrapolation, as well as of the mortar approach.

\section{A.1 Extrapolation}

The minimization interpretation of the extrapolation procedure is still valid and still leads to $\boldsymbol{M} \boldsymbol{E}=\mathcal{N}^{T} \boldsymbol{W} \mathcal{E}$. As $\boldsymbol{M}=\mathcal{N}^{T} \boldsymbol{W} \mathcal{N}$ is singular, this problem has more than a unique solution. Using a generalized inverse $\boldsymbol{M}^{+}$of $\boldsymbol{M}[17,15]$ (satisfying $\left.\boldsymbol{M} \boldsymbol{M}^{+} \boldsymbol{M}=\boldsymbol{M}\right)$ and denoting the null space of $\boldsymbol{M}$ by $\boldsymbol{R}(\boldsymbol{M R}=\mathbf{0})$, one gets:

$$
\boldsymbol{E}=\boldsymbol{M}^{+} \mathcal{N}^{T} \boldsymbol{W} \mathcal{E}+\boldsymbol{R} \boldsymbol{\alpha}
$$

This defines the new extrapolation operator $\boldsymbol{E}=\mathcal{L} \mathcal{E} . \boldsymbol{\alpha}$ are the "coordinates" in the null space $\boldsymbol{R}$, and are undefined.

The solvability condition $\boldsymbol{R}^{T} \mathcal{N}^{T} \boldsymbol{W} \mathcal{E}=0$ is automatically satisfied. Effectively, $\boldsymbol{M} \boldsymbol{R}=\mathbf{0}=\mathcal{N}^{T} \boldsymbol{W} \mathcal{N} \boldsymbol{R}$, this leads to $\boldsymbol{R}^{T} \mathcal{N}^{T} \boldsymbol{W} \mathcal{N} \boldsymbol{R}=0$; if all the integration points are such that $w_{k} J\left(M_{k}\right)>0, \boldsymbol{W}$ is SPD and thus $\boldsymbol{R}^{T} \mathcal{N}^{T}=\mathbf{0}$. One requirement for choosing the integration scheme is therefore that it has all positive weights; this is not always the case for any integration scheme [10].

Of course, the property of the extrapolation and interpolation operators changes: if $\mathcal{N}^{T}$ is surjective, $\mathcal{N}^{T} \mathcal{F}=\mathbf{0} \Rightarrow \mathcal{F}=\mathbf{0}$; then, $\boldsymbol{M} \boldsymbol{M}^{+} \boldsymbol{M}=\boldsymbol{M}$ is written $\mathcal{N}^{T} \boldsymbol{W} \mathcal{N} \boldsymbol{M}^{+} \boldsymbol{M}=\mathcal{N}^{T} \boldsymbol{W} \mathcal{N}$, so $\mathcal{N} \boldsymbol{M}^{+} \boldsymbol{M}=\mathcal{N}$ and $\mathcal{N} \boldsymbol{M}^{+} \mathcal{N}^{T} \boldsymbol{W} \mathcal{N}=$ $\mathcal{N}$. With $\mathcal{E}=\mathcal{N} \boldsymbol{E}$ (which is always possible: $\mathcal{N} \boldsymbol{E}$ spans all the space where $\mathcal{E}$ is), and since $\mathcal{N} \boldsymbol{R}=\mathbf{0}$, one gets $\mathcal{N}\left(\boldsymbol{M}^{+} \mathcal{N}^{T} \boldsymbol{W} \mathcal{E}+\boldsymbol{R} \boldsymbol{\alpha}\right)=\mathcal{N} \boldsymbol{E}=\mathcal{E}$. So, 
$\mathcal{N} \mathcal{L}=\mathbf{1}_{i}$. The new property for the consistent interpolation $\mathcal{N}$ and the corresponding extrapolation $\mathcal{L}$ is then

$$
\mathcal{N} \mathcal{L}=\mathbf{1}_{i}
$$

$\boldsymbol{\alpha}$ can be chosen by expressing an additional constraint on $\boldsymbol{E}$. For instance, minimizing the oscillation of $\boldsymbol{E}$ with respect to its mean value. In that case, if $1_{o}$ denotes the unit column vector of size $n_{o}=1_{o}^{T} 1_{o}$ (number of nodes of the element), the average field of $\boldsymbol{E}$ is $\overline{\boldsymbol{E}}=\frac{1}{n_{o}} 1_{o} 1_{o}^{T} \boldsymbol{E}$, so $\boldsymbol{E}-\overline{\boldsymbol{E}}=\boldsymbol{Q} \boldsymbol{E}$ with a symmetric projector $\boldsymbol{Q}=\mathbf{1}_{o}-\frac{1}{n_{o}} 1_{o} 1_{o}^{T}$. Minimizing the distance to the average value is $\min \boldsymbol{\alpha}(\boldsymbol{E}-\overline{\boldsymbol{E}})^{T}(\boldsymbol{E}-\overline{\boldsymbol{E}})$ and leads to $\boldsymbol{\alpha}=-(\boldsymbol{R} \boldsymbol{Q} \boldsymbol{R})^{-1} \boldsymbol{R}^{T} \boldsymbol{Q} \boldsymbol{M}^{+} \mathcal{N}^{T} \boldsymbol{W} \mathcal{E}$, therefore $\boldsymbol{E}=\boldsymbol{p} \boldsymbol{M}^{+} \mathcal{N}^{T} \boldsymbol{W} \mathcal{E}$ with a non symmetric projector

$$
p=1-\boldsymbol{R}(\boldsymbol{R} Q \boldsymbol{R})^{-1} \boldsymbol{R}^{T} \boldsymbol{Q}
$$

The extrapolation is finally $\mathcal{L}=\boldsymbol{p} \boldsymbol{M}^{+} \mathcal{N}^{T} \boldsymbol{W}$. Remark that with the use of a projector in the $\boldsymbol{R}$ direction $(\boldsymbol{p} \boldsymbol{R}=\mathbf{0})$, the result is independent of the choice of the generalized inverse $\boldsymbol{M}^{+}$. Nevertheless, other choices for $\boldsymbol{p}$ are possible, and one will be discussed in the next Section.

\section{A.2 Mortar-like prolongation}

A trivial generalization when elementary mass matrices are singular is to choose $\boldsymbol{P}_{21}=\boldsymbol{p}_{2} \boldsymbol{M}_{2}^{+} \boldsymbol{M}_{21}, \boldsymbol{R}_{12}=\boldsymbol{p}_{1} \boldsymbol{M}_{1}^{+} \boldsymbol{M}_{12}$ (with $\boldsymbol{M}_{12}=\boldsymbol{M}_{21}^{T}$ ), and as previously $\mathcal{P}_{21}=\mathcal{N}_{2} \boldsymbol{P}_{21} \mathcal{L}_{1}$ and $\boldsymbol{R}_{12}=\boldsymbol{W}_{1}^{-1} \mathcal{P}_{21}^{T} \boldsymbol{W}_{2}$. Let us recall that

$$
\begin{aligned}
\mathcal{L}_{1}=\boldsymbol{p}_{1} \boldsymbol{M}_{1}^{+} \mathcal{N}_{1}^{T} \boldsymbol{W}_{1}^{T}=\left(\boldsymbol{p}_{1} \boldsymbol{M}_{1}^{+}\right) \mathcal{N}_{1}^{T} \boldsymbol{W}_{1} \\
\mathcal{L}_{2}=\boldsymbol{p}_{2} \boldsymbol{M}_{2}^{+} \mathcal{N}_{2}^{T} \boldsymbol{W}_{2}=\left(\boldsymbol{p}_{2} \boldsymbol{M}_{2}^{+}\right) \mathcal{N}_{2}^{T} \boldsymbol{W}_{2}
\end{aligned}
$$

Then,

$$
\begin{gathered}
\mathcal{R}_{12}=\mathcal{N}_{1} \boldsymbol{R}_{12} \mathcal{L}_{2}=\mathcal{N}_{1}\left(\boldsymbol{M}_{1}^{+} \boldsymbol{p}_{1}^{T}\right) \boldsymbol{M}_{12}\left(\boldsymbol{M}_{2}^{+} \boldsymbol{p}_{2}^{T}\right) \mathcal{N}_{2}^{T} \boldsymbol{W}_{2} \\
\mathcal{P}_{12}=\mathcal{N}_{1} \boldsymbol{P}_{12} \mathcal{L}_{2}=\mathcal{N}_{1}\left(\boldsymbol{p}_{1} \boldsymbol{M}_{1}^{+}\right) \boldsymbol{M}_{12}\left(\boldsymbol{p}_{2} \boldsymbol{M}_{2}^{+}\right) \mathcal{N}_{2}^{T} \boldsymbol{W}_{2}
\end{gathered}
$$

To recover symmetry of the formulation, i.e. $\boldsymbol{\mathcal { R }}_{12}=\mathcal{P}_{12}, \boldsymbol{p}$ and $\boldsymbol{M}^{+}$should satisfy $\boldsymbol{M}^{+} \boldsymbol{p}^{T}=\boldsymbol{p} \boldsymbol{M}^{+}$for each mesh. This is not the case with the previous projector $\boldsymbol{p}$.

The simplest choice for the projector $\boldsymbol{p}$ such that $\boldsymbol{p} \boldsymbol{M}^{+}$is symmetric, is (i) to select an invertible generalized inverse $\boldsymbol{M}^{+}$(Moore-Penrose is not, but there are always some other; for instance $\boldsymbol{M}^{+}=\left(\boldsymbol{M}+\beta \boldsymbol{R} \boldsymbol{R}^{T}\right)^{-1}$ with $\left.\beta>0\right)$; (ii) to set $\boldsymbol{Q}=\left(\boldsymbol{M}^{+}\right)^{-1}$ in the expression (15) of $\boldsymbol{p}$. Indeed, $\boldsymbol{p} \boldsymbol{M}^{+}=\boldsymbol{M}^{+}{ }_{-}$ $\boldsymbol{R}\left(\boldsymbol{R}^{T} \boldsymbol{Q R}\right)^{-1} \boldsymbol{R}^{T}$ is then symmetric.

The new interpretation of $\boldsymbol{p}$ arises from $\min _{\boldsymbol{\alpha}} \boldsymbol{E}^{T}\left(\boldsymbol{M}^{+}\right)^{-1} \boldsymbol{E}: \boldsymbol{\alpha}$ is found to minimize the $\left(\boldsymbol{M}^{+}\right)^{-1}$-norm of $\boldsymbol{E}$. This constraint on $\boldsymbol{E}$ allows to get a symmetric formulation.

The choice of a sensible regular generalized inverse, and comparison tests are still to be done in this case. 